ARTICLE

DOI: $10.1038 / s 41467-017-00153-y$

\title{
In vivo cation exchange in quantum dots for tumor-specific imaging
}

Xiangyou Liu ${ }^{1,2}$, Gary B. Braun ${ }^{1}$, Mingde Qin ${ }^{3}$, Erkki Ruoslahti ${ }^{1,4}$ \& Kazuki N. Sugahara ${ }^{1,2}$

In vivo tumor imaging with nanoprobes suffers from poor tumor specificity. Here, we introduce a nanosystem, which allows selective background quenching to gain exceptionally tumor-specific signals. The system uses near-infrared quantum dots and a membraneimpermeable etchant, which serves as a cation donor. The etchant rapidly quenches the quantum dots through cation exchange (ionic etching), and facilitates renal clearance of metal ions released from the quantum dots. The quantum dots are intravenously delivered into orthotopic breast and pancreas tumors in mice by using the tumor-penetrating iRGD peptide. Subsequent etching quenches excess quantum dots, leaving a highly tumor-specific signal provided by the intact quantum dots remaining in the extravascular tumor cells and fibroblasts. No toxicity is noted. The system also facilitates the detection of peritoneal tumors with high specificity upon intraperitoneal tumor targeting and selective etching of excess untargeted quantum dots. In vivo cation exchange may be a promising strategy to enhance specificity of tumor imaging.

\footnotetext{
${ }^{1}$ Cancer Research Center, Sanford Burnham Prebys Medical Discovery Institute, 10901 N Torrey Pines Road, La Jolla, California 92037, USA. ${ }^{2}$ Department of Surgery, Herbert Irving Comprehensive Cancer Center, Columbia University College of Physicians and Surgeons, 1130 St Nicholas Avenue, New York, New York 10032, USA. ${ }^{3}$ Program of Materials Science and Engineering, University of California, San Diego, 9500 Gilman Drive, La Jolla, California 92093 , USA. ${ }^{4}$ Center for Nanomedicine and Department of Cell, Molecular and Developmental Biology, University of California, Santa Barbara, Santa Barbara, California 93106, USA. Correspondence and requests for materials should be addressed to G.B.B. (email: gbraun5468@gmail.com) or to K.N.S. (email: sugahara@sbpdiscovery.org)
} 
T he design of nanoprobes for in vivo tumor imaging has traditionally focused on optimization of probe properties such as size, surface coating, and signal intensity to maximize target sensitivity and specificity ${ }^{1,2}$. Nanoparticles larger than the renal filtration threshold $(\sim 6 \mathrm{~nm})$ circulate longer and accumulate more efficiently in tumors than small molecules. However, long washout periods increase background signals especially in the mononuclear phagocyte system (MPS; e.g., liver, spleen). Although surface modification with polyethylene glycol (PEG) reduces non-specific uptake by liver Kupffer cells, the tumor to liver ratio $(\mathrm{T} / \mathrm{Li})$ for nanoparticles that are not cleared through the kidneys generally decreases with time, leading to degraded image contrast ${ }^{3-5}$. In this study, we explore an alternative strategy to enhance tumor specificity-selective elimination of background signals while preserving tumor signals in vivo. We utilize photoluminescent quantum dots (QDs) as the platform for quenchable nanoprobes based on the ability of QDs to undergo cation exchange (ionic etching) with external metal ions.

Cation exchange in QDs allows rapid modification of the elemental composition and crystal structure, and has been exploited to synthesize new nanostructures and modify photoluminescence (PL) characteristics 6, 7. In QD cation exchange, metal cations that are embedded within an anion lattice can exchange with free metal ions in solution. In particular, in QDs built from large polarizable sulfide $\left(\mathrm{S}^{2-}\right)$, selenide $\left(\mathrm{Se}^{2-}\right)$, or phosphorus $\left(\mathrm{P}^{3-}\right)$, the internal cations can pass through open sites between anions leading to effective cation exchange. Notably, the anionic framework and geometry of the QD core may be preserved during cation exchange ${ }^{6}$.

Here, we introduce a biocompatible QD platform, which loses PL upon cation exchange, and achieves tumor-specific in vivo imaging in 3 steps: First, active delivery of the QDs into extravascular tumor tissue and cells to gain bright tumor a

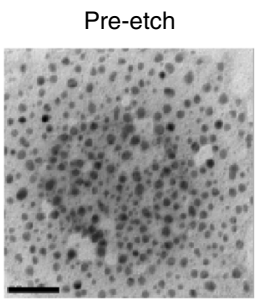

d

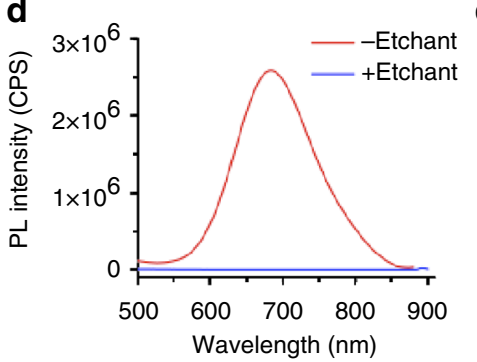

h

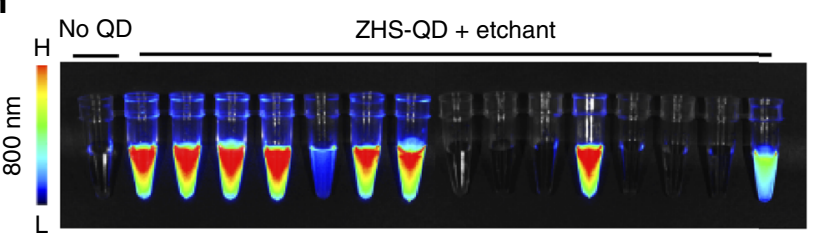

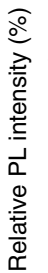
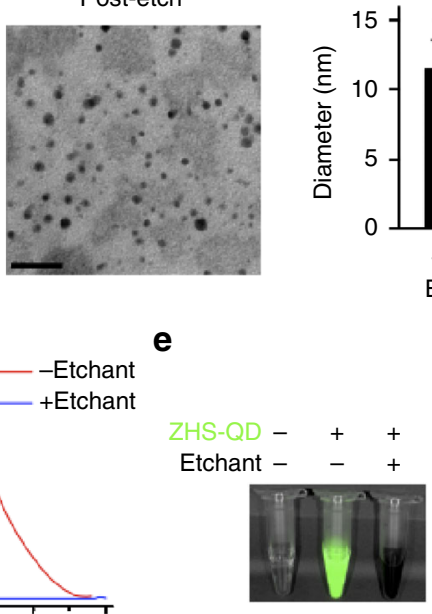
e

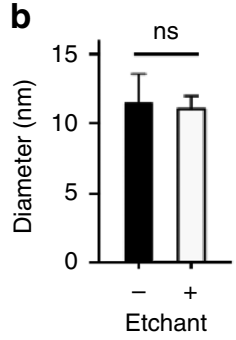

f c

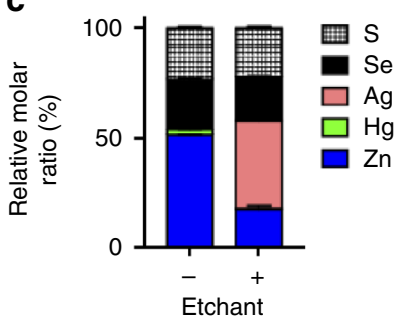

g

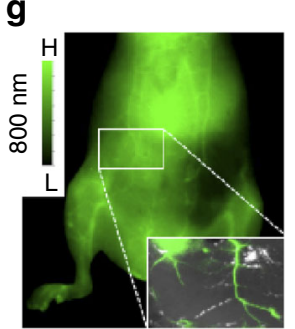

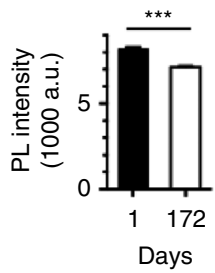

i

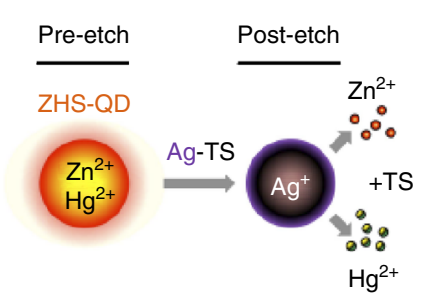

j

- Etchant $\mathbf{\square}+$ Etchant

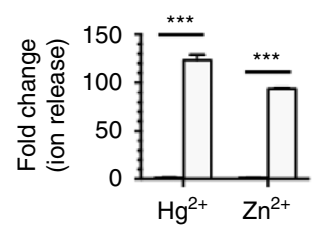

Fig. 1 Characterization of etchable ZHS-QDs. $\mathbf{a}, \mathbf{b}, \mathbf{d}$ TEM images $\mathbf{a}$ hydrodynamic diameters $\mathbf{b}$ and PL spectra at $450 \mathrm{~nm}$ excitation $\mathbf{d}$ of ZHS-QDs before and after Ag-TS treatment. $n=6$ per group; Scale bars, $50 \mathrm{~nm}$. c EDS analysis of ZHS-QDs before and after Ag-TS treatment. Refer to Supplemental Table 1 for complete data set. $n=3$ per group. e NIR image of ZHS-QDs (green) with and without Ag-TS treatment taken with a Li-Cor Pearl imager under an $800 \mathrm{~nm}$ channel. $\mathbf{f}$ NIR signals of ZHS-QDs quantified at days 1 and 172 post-synthesis. $n=5$ per group. $\mathbf{g}$ NIR images of the ventral side of a mouse intravenously injected with ZHS-QDs. Inset, ex vivo NIR image of the skin. $\mathbf{h}$ In vitro etching of ZHS-QDs by various chemicals. NIR images (upper panel) and the emission intensity of the ZHS-QDs before and after etching (bottom panel, $n=4$ per group) are shown. Each column corresponds to the tube above. i Schematic of ZHS-QD etching. Ag-TS quenches ZHS-QDs by providing $\mathrm{Ag}^{+}$in exchange with $\mathrm{Zn}^{2+}$ and $\mathrm{Hg}^{2+}$. $\mathbf{j} \mathrm{Hg}$ and $\mathrm{Zn}$ released from ZHS-QDs with and without Ag-TS treatment. Columns represent fold over non-etched group. $n=3$ per group. Statistics, Student's $t$-test $\mathbf{b}, \mathbf{f}, \mathbf{j}$ or one-way analysis of variance $\mathbf{h}$; error bars, SEM; ns, not significant; ${ }^{\star \star} P<0.01$; ${ }^{\star \star \star} P<0.001$ 


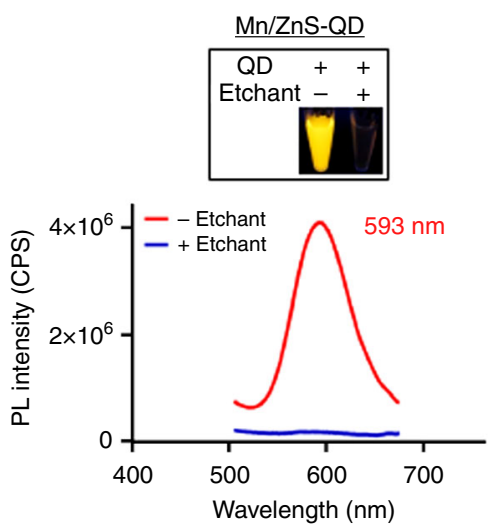

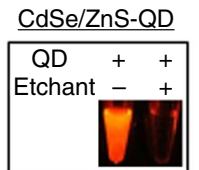

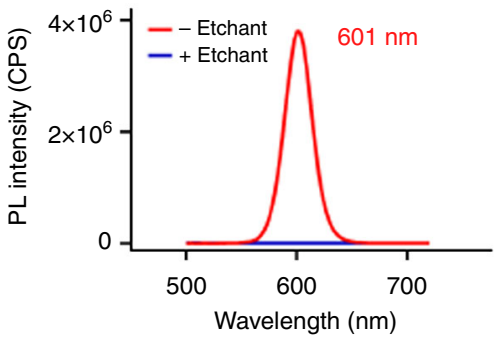

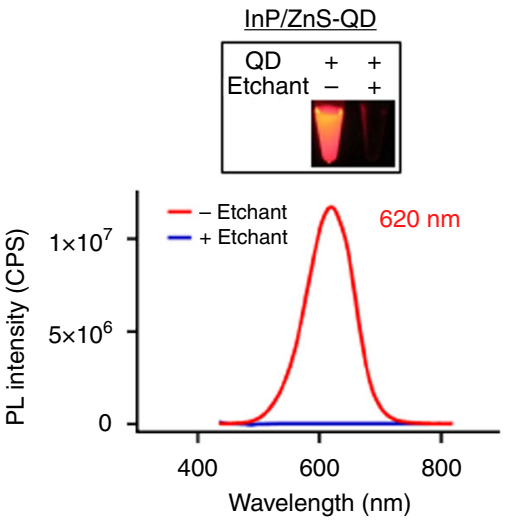

CIS/ZnS-QD

with amphiphilic coating
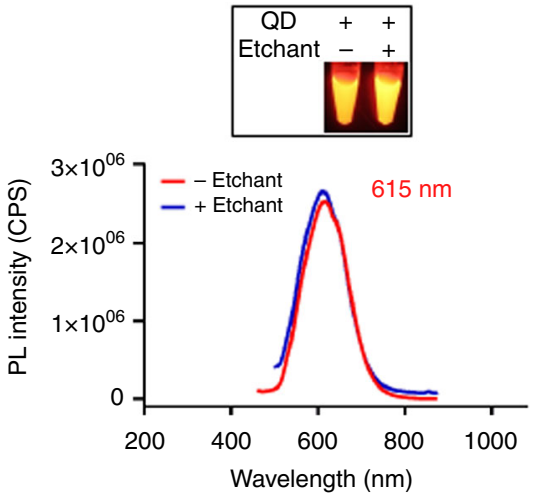

Fig. 2 In vitro etching of various QDs with Ag-TS. PL spectra of the QDs before (red) and after (blue) etching are shown. The emission peaks of the QDs are also listed. The boxed panels show fluorescence images of the QD aqueous solutions with or without etching. QDs synthesized in-house (i.e., Mn/ZnS-QD) and high-quality commercial QDs (i.e., CdSe/ZnS-QD, InP/ZnS-QD, and CIS/ZnS-QD) were each etchable by Ag-TS. The Mn/ZnS-QD, $\mathrm{InP} / \mathrm{ZnS}$ - and CIS/ZnS-QDs had coatings of 3-mercaptopropionic acid. Amphiphiliccoating with $\mathrm{HS}-\left(\mathrm{CH}_{2}\right)_{11}-\left(\mathrm{OCH}_{2} \mathrm{CH}_{2}\right)_{6}-\mathrm{NH}_{2}$ prevented CIS/ZnS-QD from being etched

signals. Second, induction of cation exchange in excess QDs remaining in the circulation to quench background signals. Third, effective renal excretion of the cations released from the QDs to minimize potential toxicity. The platform also probes peritoneal tumors with high specificity when delivered through the abdominal cavity suggesting its potential role as an aid in the diagnosis and surgery for peritoneal carcinomatosis.

\section{Results}

Synthesis of PEGylated near-infrared QDs. We first synthesized highly dispersed near-infrared (NIR) $\mathrm{Zn}_{x} \mathrm{Hg}_{1-x} \mathrm{Se}_{y} \mathrm{~S}_{1-y}$ QDs (ZHS-QDs) consisting of zinc $\left(\mathrm{Zn}^{2+}\right)$, mercury $\left(\mathrm{Hg}^{2+}\right), \mathrm{Se}^{2-}$ and $\mathrm{S}^{2-} \cdot \mathrm{Hg}^{2+}$ was doped into the core as a tracer to accurately study tissue distribution and clearance kinetics. The QDs were coated with PEG to reduce MPS uptake ${ }^{4}$. Transmission electron microscopy (TEM) revealed a core diameter of $6.6 \pm 2.3 \mathrm{~nm}$ (mean \pm standard deviation; Fig. 1a and Supplementary Fig. 1a). Dynamic light scattering (DLS) showed a hydrodynamic diameter of $\sim 12 \mathrm{~nm}$ (Fig. 1b), a size above the renal filtration threshold ${ }^{8}$. Elemental analysis by inductively coupled plasma optical emission spectroscopy (ICP-OES) and energy-dispersive X-ray spectroscopy (EDS) confirmed the composition of the QDs (Fig. 1c and Supplementary Fig. 1b). PEG was detected by EDS as carbon (C) and oxygen (O), which accounted for $\sim 90 \%$ of the total atoms (Supplementary Table 1). The PL emission peak was at $685 \mathrm{~nm}$, which was consistent under different excitation wavelengths (Fig. 1d and Supplementary Fig. 1c). The quantum yield (QY) was $12 \%$ based on a calculation using Rhodamine $6 \mathrm{G}$ as a standard. A strong PL signal at $800 \mathrm{~nm}$ (the tail of the emission peak) was obtained using 785-nm excitation (Fig. 1e and Supplementary Fig. 1d), the preferred excitation wavelength for a Li-Cor Pearl Impulse imager to minimize background signals during in vivo imaging (Supplementary Fig. 1e). Approximately $90 \%$ of the PL intensity remained after the QDs were stored at $4{ }^{\circ} \mathrm{C}$ for 6 months without visible aggregation of the QDs (Fig. 1f). Intravenous injection of the ZHS-QDs provided strong NIR vascular signals in mice (Fig. 1g). Additional QD properties are summarized in Supplementary Table 2.

In vitro cation exchange reactions in ZHS-QDs. We next developed an 'etchant', which serves as a cation donor to the QDs and quenches the PL of the QDs by cation exchange. Various chemicals with cation-exchange capacity quenched ZHS-QDs in vitro except for $\mathrm{Zn}(\mathrm{OAc})_{2}$ (zinc acetate dehydrate), which increased the PL probably by providing extra $\mathrm{Zn}^{2+}$ to the QDs (Fig. 1h). One of the most efficient etchants was $\mathrm{Ag}\left(\mathrm{S}_{2} \mathrm{O}_{3}\right)_{2}{ }^{3-}$ (Ag-TS), which consisted of silver ions $\left(\mathrm{Ag}^{+}\right)$stabilized with thiosulfate (TS), a metal chelator used as a clinical detoxifying agent for heavy metal ions ${ }^{9,10}$. Ag-TS provided $\mathrm{Ag}^{+}$to the QDs in exchange with $\mathrm{Zn}^{2+}$ and $\mathrm{Hg}^{2+}$ (Fig. 1i), and rapidly quenched the QDs (Fig. 1d,e). Stoichiometric in vitro quenching studies in aqueous solution at room temperature showed that $>95 \%$ of ZHS-QDs were quenched when Ag-TS was added at an Ag to $\mathrm{Zn}$ molar ratio of 1:1 or higher (Supplementary Fig. 1f). Treating 
a
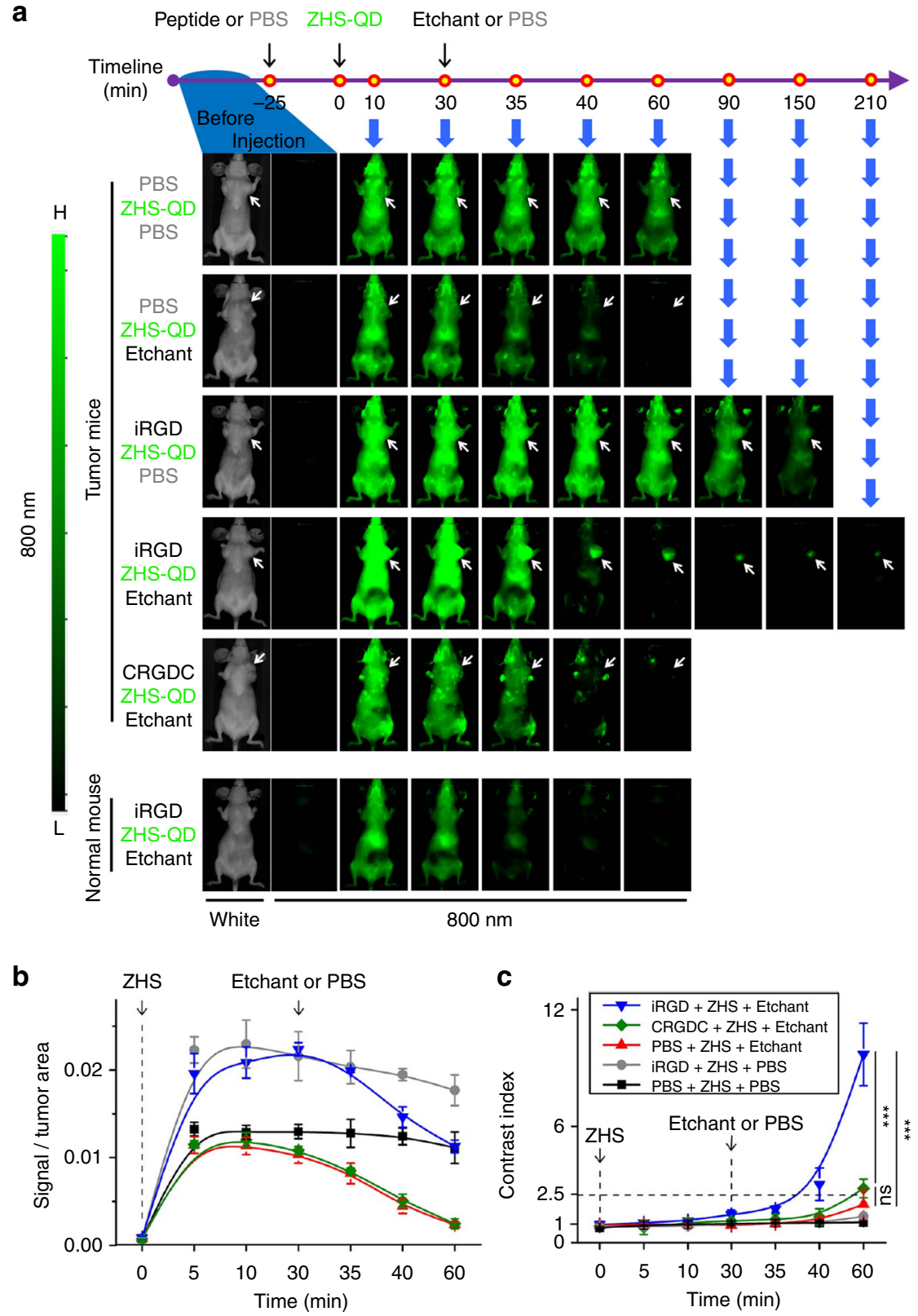

Fig. 3 Time-dependent in vivo breast tumor imaging with etchable ZHS-QDs. Normal mice and mice bearing orthotopic MCF10CA1a human breast tumors received an intravenous injection of $\mathrm{R} G \mathrm{D}, \mathrm{CRGDC}$, or PBS before intravenous ZHS-QD injection. Ag-TS or PBS was given intraperitoneally. $n=4$ per group. a The mice were anesthetized and imaged from the ventral side with a Li-Cor Pearl imager under $800 \mathrm{~nm}$ channel at the indicated time points. Arrows, tumors. b NIR signals in the tumor per unit area plotted against time. c Time-dependent changes of $\mathrm{Cl}$ in the tumor area. Statistics, two-way analysis of variance; error bars, SEM; ns, not significant; ${ }^{\star \star \star} P<0.001$

ZHS-QDs with Ag-TS (Ag to $\mathrm{Zn}=1: 1)$ resulted in near complete quenching of the QDs within 10 s (Supplementary Fig. 1g). These results are consistent with earlier studies, which reported rapid and nearly complete cation exchange reactions under ambient conditions $^{6,}{ }^{11}$. The completion of cation exchange can be attributed to several factors: adequate $\mathrm{Ag}$ to $\mathrm{Zn}$ molar ratio, the small number of atomic layers and large surface area of QDs, the high thermodynamic driving force to form the exchanged product $\left(\mathrm{Ag}_{2} \mathrm{~S}\right.$ and $\left.\mathrm{Ag}_{2} \mathrm{Se}\right)$, and the TS solubilizing agent, which ligates free $\mathrm{Ag}^{+}, \mathrm{Zn}^{2+}$ and $\mathrm{Hg}^{2+}$ ions to minimize the formation of any surface nanoprecipitate that might otherwise reduce exchange rates ${ }^{7}$.

ICP-OES and EDS of the QDs showed that Ag-TS treatment led to the release of $\mathrm{Hg}^{2+}$ and $\mathrm{Zn}^{2+}$ from the core in exchange for
$\mathrm{Ag}^{+}$(Fig. 1c and Supplementary Fig. 1b). The released $\mathrm{Hg}^{2+}$ and $\mathrm{Zn}^{2+}$ were detected in the buffer by ICP-OES (Fig. 1j). These results confirmed that effective cation exchange was achieved. More than $80 \%$ of the $\mathrm{C}$ and $\mathrm{O}$ remained after Ag-TS treatment suggesting minimal change in the surface PEG layer (Supplementary Table 1). The XRD spectra of ZHS-QDs after $\mathrm{Ag}$-TS treatment showed that new peaks corresponding to $\mathrm{Ag}_{2} \mathrm{~S}$ and $\mathrm{Ag}_{2} \mathrm{Se}$ appeared confirming the ICP-OES and EDS results (Supplementary Fig. 1h). Accordingly, the extinction coefficient of the QDs increased after etching, consistent with Ag-TS inducing a material transformation of the core (Supplementary Table 2). The QD core size after etching was $6.0 \pm 1.9 \mathrm{~nm}$ suggesting a small change in the size of the QDs (Fig. 1a and 
a

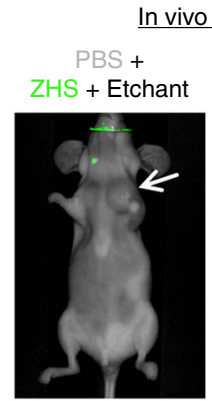

In vivo imaging

iRGD +

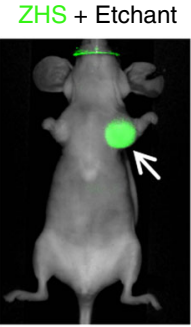

b In situ imaging

$\mathrm{iRGD}+\mathrm{ZHS}+$ Etchant

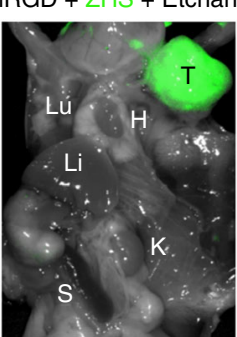

C

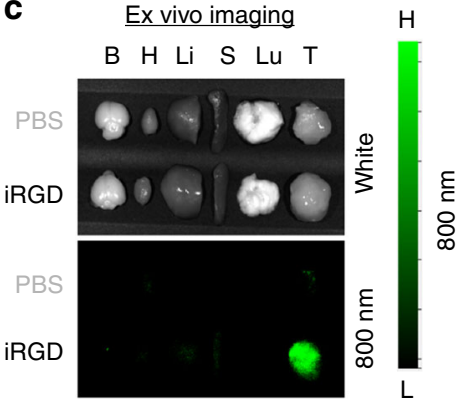

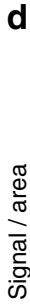

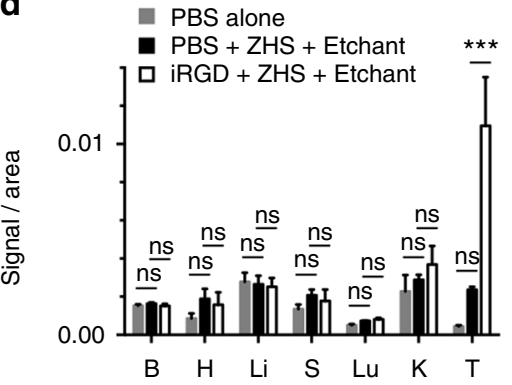

f

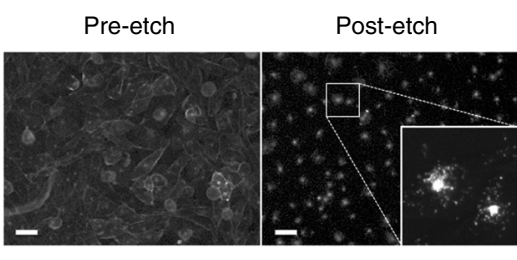

e $P B S+Z H S-Q D$
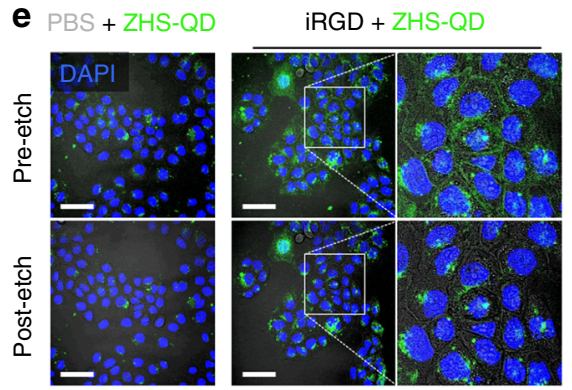

g $P B S+$

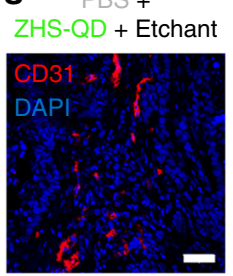

$i R G D+Z H S-Q D+$ Etchant

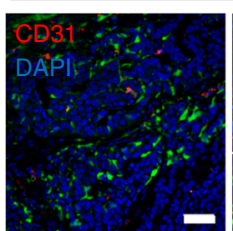

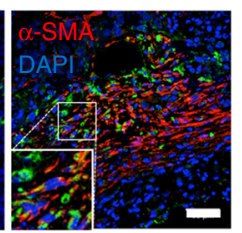

Fig. 4 Breast tumor-specific imaging achieved by extravascular delivery of etchable ZHS-QDs. Mice bearing orthotopic MCF10CA1a human breast tumors received an intravenous injection of $\mathrm{R}$ RGD or PBS before an intravenous dose of ZHS-QDs. Ag-TS was given intraperitoneally. $n=4$ per group. a The mice were anesthetized and imaged with a Li-Cor Pearl imager 40 min after etching. Arrows, tumors. $\mathbf{b}$ In situ NIR imaging of the mice after euthanasia and necropsy performed under deep anesthesia. c NIR images of collected tissues. d NIR signal per unit area in collected tissues (left panel) and T/Li ratio (right panel). B, brain; $\mathrm{H}$, heart; Li, liver; S, spleen; Lu, lung; $\mathrm{K}$, kidney; T, tumor. Statistics, two-way analysis of variance (left panel) or Student's $t$-test (right panel); error bars, SEM; ns, not significant; ${ }^{\star} P<0.05$; ${ }^{\star \star \star} P<0.001$. e Confocal micrographs of cultured MCF10CA1a cells treated with ZHS-QDs with or without free iRGD followed by etching with Ag-TS. Note that the QDs were internalized into the cells in the presence of iRGD, and that only the extracellular QDs were etched. Blue, Hoechst 33342; green, ZHS-QDs; scale bars, $50 \mu \mathrm{m}$. Insets show a magnified view of the boxed areas. $\mathbf{f}$ Epifluorescence micrographs of cultured PC-3 human prostate cancer cells treated with cell-penetrating QDs followed by etching with Ag-TS. PC-3 cells were incubated with CdSe/ZnS QDs coated with a cell-penetrating peptide KCDGRPARPAR. Only speckled peri-nuclear signals remain after etching. Scale bars, 50 um. $\mathbf{g}$ The tumors shown in $\mathbf{c}$ were processed for immunofluorescence staining and subjected to confocal microscopy. Blue, DAPI; red, CD31 or $\alpha-S M A ;$ green, ZHS-QDs; scale bars, $50 \mu \mathrm{m}$. The boxed area is magnified. Note that the QD signals are found in the perinuclear area of the cells

Supplementary Fig. 1a). The hydrodynamic size also remained unchanged and precipitation was not observed (Fig. 1b).

In vitro quenching of various QDs induced by Ag-TS. Ag-TS quenched QDs with differences in PL emission peak, QY, and composition, such as manganese-doped zinc sulfide ( $\mathrm{Mn} / \mathrm{ZnS}$ ) $\mathrm{QDs}(\mathrm{QY}=7 \%)^{12}$, cadmium selenide zinc sulfide (CdSe/ZnS) core/shell QDs(QY $=45 \%)$, indium phosphide zinc sulfide (InP/ZnS) QDs(QY $=40 \%)$, and copper indium sulfide zinc sulfide (CIS/ZnS) QDs (QY = 15\%; Fig. 2). Of note, amphiphilic coating with $\mathrm{HS}-\left(\mathrm{CH}_{2}\right)_{11}-\left(\mathrm{OCH}_{2} \mathrm{CH}_{2}\right)_{6}-\mathrm{NH}_{2}$ made CIS/ZnS-QDs resistant to Ag-TS treatment likely because the coating inhibited cation penetration. These results suggest the wide applicability of chemical etching to various types of QDs, and a way to produce etching-resistant QDs. In addition, combination of QDs with different colors and etchable properties may allow multicolor and multiplex in vivo imaging.

In vivo cation exchange reactions in ZHS-QDs. Intravenous injection of Ag-TS into mice eliminated systemic ZHS-QD signals within $30 \mathrm{~s}$ (Supplementary Fig. 2a). The etchant was quickly excreted or functionally consumed because a second dose of ZHS-QDs 10 min later produced strong signals. A second dose of Ag-TS effectively quenched the QDs indicating that repetitive in vivo imaging can be performed over short intervals. Intraperitoneal injection of Ag-TS completely eliminated QD signals in $\sim 30$ min reflecting the absorption kinetics of intraperitoneal Ag-TS into the blood stream ${ }^{13}$ (Fig. $3 a$ and Supplementary Fig. 2b). Delaying the timing of intraperitoneal Ag-TS injection allowed the QDs to enter the liver in higher quantity, and led to a significant liver signal after etching (Supplementary Fig. 2c,d). The resistance of the liver signals to Ag-TS treatment suggested that some QDs were phagocytosed by Kupffer cells, which protected the QDs from the circulating etchant. The liver signals became more evident when we modified the surface of ZHS-QDs with positive charge, which makes nanoparticles more prone to uptake by Kupffer cells ${ }^{14}$ (Supplementary Fig. 2e). Subcutaneous etchant injections were also effective. Three consecutive subcutaneous injections of a mixture of penicillamine, a clinical metal chelator ${ }^{15,16}$, and $\mathrm{CuSO}_{4}$, which efficiently etched ZHS- 
QDs in vitro (Fig. 1h), led to near-complete in vivo quenching of systemic ZHS-QD signals in 20 min (Supplementary Fig. 2f).

Elemental analysis of biological samples collected from mice that received an intravenous ZHS-QD injection revealed progressive $\mathrm{Hg}^{2+}$ shift from the blood into the liver and spleen, indicating MPS uptake of the QDs (Supplementary Fig. 3a). Subsequent etching with intraperitoneal Ag-TS caused rapid redirection of $\mathrm{Hg}^{2+}$ into the kidney and urine, apparently as a result of in vivo cation exchange and renal clearance of $\mathrm{Hg}^{2+}$. The liver $\mathrm{Hg}^{2+}$ level in the etched group remained as high as that in the non-etched group up to $24 \mathrm{~h}$ likely reflecting the QDs that had already entered the liver by that point combined with uptake of free $\mathrm{Hg}^{2+}$ (a product of etching) by hepatocytes ${ }^{17}$. However, the level gradually decreased overtime in contradiction to the progressive increase observed in the non-etched group. $\mathrm{Hg}^{2+}$ became nearly undetectable in all the organs tested within 10 days of etching. ICP-OES analysis of ZHS-QDs isolated from the serum of the mice showed that the Ag-TS treatment caused a decrease in $\mathrm{Zn}$, near complete elimination of $\mathrm{Hg}$, and new incorporation of $\mathrm{Ag}$ in the QDs supporting the notion that in vivo cation exchange occurred (Supplementary Fig. 3b). Biochemical analysis of mouse blood samples $24 \mathrm{~h}$ and 1 week after ZHS-QD and/or Ag-TS injections did not show elevated transaminases or hyperbilirubinemia suggesting minimal hepatotoxicity (Supplementary Fig. 3c). ZHS-QDs did not cause renal impairment. A possible transient creatinine increase was associated with Ag-TS injections, but it was statistically insignificant. No microscopic pathological changes were found (Supplementary Fig. 3d).

Passive distribution of ZHS-QDs into tumors. Circulating nanoparticles can passively distribute into tumors by enhanced permeability and retention (EPR) effect ${ }^{18}$. However, this process requires several hours, and passive distribution may not be enough to deliver compounds in sufficient quantity against high interstitial fluid pressure in tumors ${ }^{18,19}$. In our experiments, intravenous injection of PBS and ZHS-QDs followed by intraperitoneal injection of Ag-TS resulted in minimal tumor signals in mice bearing orthotopic MCF10CA1a human breast cancer xenograft tumors (Fig. 3a).

Tumor-specific systemic delivery of etchable ZHS-QDs. To actively deliver ZHS-QDs to tumors, we used the tumorpenetrating cyclic peptide, iRGD (amino acid sequence: CRGDKGPDC $)^{20}$. iRGD carries a tumor-specific RGD motif and an RXXR/K motif (CendR motif). The RGD motif initially targets the peptide to tumor vasculature by binding to $\alpha \mathrm{v}$ integrins, and after a proteolytic cleavage step, the CendR motif interacts with neuropilin-1. The interaction with neuropilin-1 increases extravasation and energy-dependent endocytosis of the peptide and bystander molecules in a tumor-specific manner ${ }^{20-22}$. The bystander effect peaks in about $30 \mathrm{~min}$ and remains for up to $60 \mathrm{~min}$, providing a window to deliver co-injected free compounds into the extravascular tissue in a tumor-specific manner ${ }^{23}$.

ZHS-QDs were intravenously injected into MCF10CA1a breast tumor mice 25 min after an intravenous dose of iRGD, which led to a strong tumor signal buried in systemic background signals (Fig. 3a). The tumor signal became more visible as the background gradually faded over time likely because of QD clearance by MPS or quenching by various endogenous factors such as oxidation ${ }^{24-26}$. However, the signal to noise ratio remained low (Fig. 3c). In contrast, etching with intraperitoneal Ag-TS performed $30 \mathrm{~min}$ after ZHS-QD injection steeply lowered the background signals making the tumor-specific signal become apparent (Fig. 3a). The background signals decreased to $\sim 10 \%$ within 10 min from etching, while $75 \%$ of the tumor signal remained (Fig. 3b). After $30 \mathrm{~min}$, almost no background was noted, while over $50 \%$ of the tumor signal remained. The tumorspecific signal stayed apparent for at least $210 \mathrm{~min}$ after etching. CRGDC, a control RGD peptide without tissue-penetrating properties $^{20,27}$, did not enhance tumor signals. Etching decreased tumor signals to a similar degree in each group indicating baseline passive diffusion of Ag-TS into the tumors regardless of peptide pre-injection.

Contrast index (CI), defined as equation (1), is a parameter for tumor-specific image quality ${ }^{3,28}$.

$$
\mathrm{CI}=\frac{\text { fluorescence intensity of tumor area-autofluorescence }}{\text { fluorescence intensity of normal contralateral region-autofluorescence }}
$$

A CI of 2.5 is a general cut-off for substantial tumor-specific detection with optical imaging ${ }^{3}$. In breast tumor mice that received iRGD, ZHS-QDs, and Ag-TS, CI reached 2.5 at $10 \mathrm{~min}$ post-etching, followed by a continuous increase to reach 10 after an additional $20 \mathrm{~min}$ (Fig. 3c). Mice that received PBS or CRGDC pre-injection instead of iRGD reached a CI of $\sim 2.5$ after etching, representing the EPR effect. However, the dim tumor signals were suboptimal for practical imaging.

The quality of breast tumor images was further assessed at $40 \mathrm{~min}$ post-etching. In vivo live animal imaging (Fig. 4a), in situ imaging after necropsy (Fig. 4b), and ex vivo imaging of resected tissues (Fig. 4c) all showed tumor-specific signals in the iRGD group, while minimal fluorescence was found in the non-iRGD group. Signal intensity in the resected tumors was fivefold higher in the iRGD group than the control (Fig. 4d). T/Li ratio, a hallmark for tumor specificity, was 4.9 in the iRGD group, which is 17.5 -fold higher than the average value (0.28) of previously reported inorganic nanoprobes in general, and 70 -fold higher than the average value $(0.07)$ of those with a size similar to the ZHS-QDs ${ }^{3,5}$ (Fig. 4d). The T/Li ratio in the non-iRGD group was 0.98 , suggesting near-equal $\mathrm{QD}$ intensity in the tumor and the liver.

Effects of etching on extracellular and endocytosed QDs. These results indicate successful tumor-specific extravascular delivery of ZHS-QDs by iRGD, and effective intravascular etching by Ag-TS. However, given that Ag-TS can also extravasate to some extent through leaky tumor blood vessels (Fig. 3b) ${ }^{29}$, we hypothesized that the QDs in the extravascular tumor tissue were internalized in cells and that the etching was limited to the extracellular space. In a cellular context, neuropilin-binding CendR peptides such as iRGD activate an endocytic pathway similar to macropinocytosis, which helps bystander compounds to enter cells ${ }^{22}$. On the other hand, Ag-TS has an overall negative charge and hardly diffuses across cell membranes, making this hypothesis a valid one. In in vitro cell culture studies, iRGD allowed co-applied ZHS-QDs to enter MCF10CA1a cells (Fig. 4e). Addition of Ag-TS to the culture media led to etching of membrane-bound QDs leaving internalized QDs intact indicating that the etching was limited to the extracellular space. Similar results were obtained when Ag-TS was applied to PC-3 human prostate cancer cells pre-cultured in the presence of CdSe/ZnS-QDs coated with RPARPAR, a neuropilin-1-binding CendR peptide, with an $\mathrm{N}$-terminal KCDG peptide linker (Fig. 4f ${ }^{30}$. Confocal microscopy of MCF10CA1a tumor sections collected from mice, which received iRGD, ZHS-QDs, and Ag-TS, showed widespread ZHS-QDs in the extravascular tumor space (Fig. 4g). The majority of the QDs were internalized into tumor cells and also stromal cells expressing $\alpha$-smooth muscle actin ( $\alpha$-SMA), a marker for carcinoma-associated fibroblasts (CAFs). Only minor QD signals were noted in control organs (Supplementary Fig. 4a). 


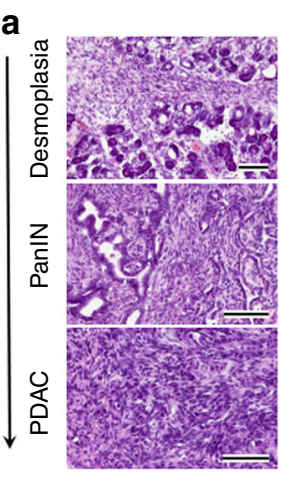

d

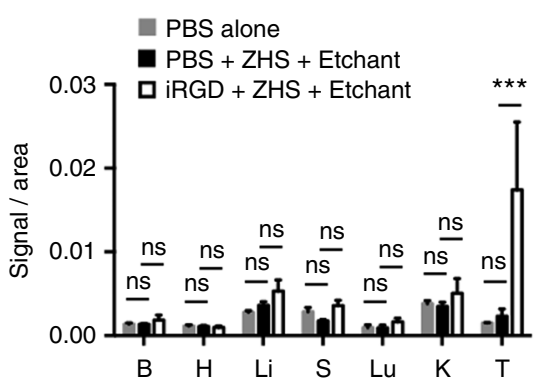

b

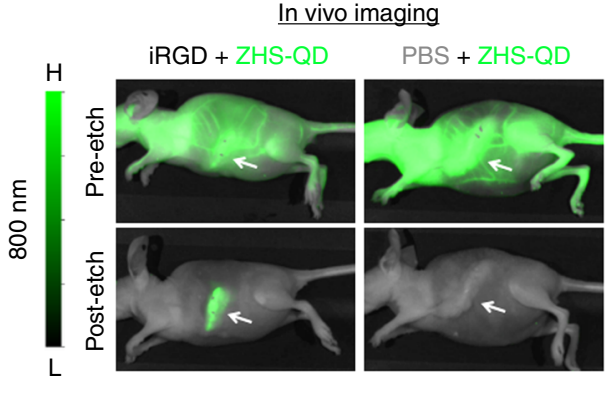

C

Ex vivo imaging

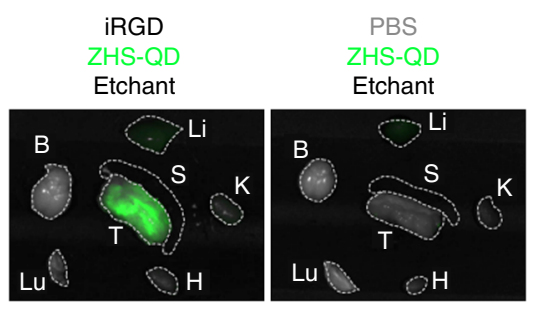

e
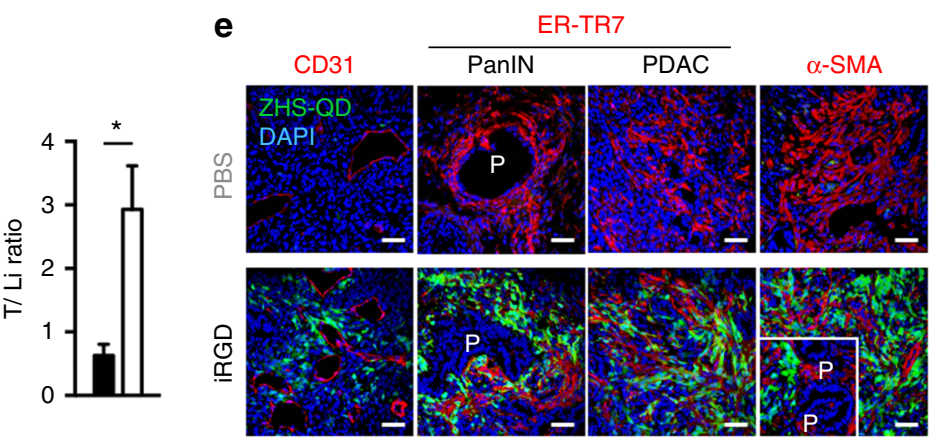

Fig. 5 Desmoplastic PDAC imaging with etchable ZHS-QDs. a H\&E staining of orthotopic KRAS-Ink tumor tissue collected from mice. A mixed histology ranging from pancreas tissue with severe desmoplasia, high-grade PanIN, to full-blown PDAC is observed. $n=3$, scale bars, $100 \mu \mathrm{m}$. b-e Mice bearing orthotopic KRAS-Ink PDAC tumors received intravenous injections of iRGD or PBS 25 min before ZHS-QD injection. Ag-TS was intravenously injected 30 min after the QD injection. $n=3$ per group. NIR images of anesthetized mice $\mathbf{b}$ and collected tissues $\mathbf{c}$. Arrows, tumors; dotted lines, tissues. $\mathrm{B}$, brain; $\mathrm{H}$, heart; Li, liver; S, spleen; Lu, lung; K, kidney; T, tumor. NIR signal per unit area in collected tissues (left panel) and T/Li ratio (right panel) d. Statistics, two-way analysis of variance (left panel) or Student's $t$-test (right panel); error bars, SEM; ns, not significant; ${ }^{\star} P<0.05$; ${ }^{\star \star \star} P<0.001$.

Confocal micrographs of tumor sections e. Blue, DAPI; red, CD31, ER-TR7 or $\alpha$-SMA; green, ZHS-QDs; scale bars, $50 \mu \mathrm{m}$; P stands for PanIN. Inset, an area with PanINs

These results support the hypothesis that the QDs were delivered into cells in the extravascular tumor tissue, which made them resistant to subsequent etching.

In vivo imaging of pancreatic cancer with ZHS-QDs. The finding that the iRGD/ZHS-QD system targeted CAFs prompted us to test the utility of the system for imaging desmoplastic pancreatic ductal adenocarcinoma (PDAC). We prepared mice bearing orthotopic tumors created with KRAS-Ink PDAC cells established from transgenic $p 48$-CRE/LSL-KrasG12D/INK4a flox mice $^{31,32}$. The tumors develop a mixture of high-grade pancreatic intraepithelial neoplasia (PanIN) and desmoplastic PDAC $^{32}$ (Fig. 5a). Injection of ZHS-QDs and Ag-TS resulted in minimal signals (Fig. 5b). Adding iRGD pre-injection led to a strong focal signal in the left flank where the tumor was located. Ex vivo imaging confirmed a strong tumor-specific signal (Fig. 5c). The $\mathrm{T} / \mathrm{Li}$ ratio was $2.9, \sim 10$ times higher than previously reported values of general inorganic nanoprobes ${ }^{3}$ (Fig. 5d). The QDs widely distributed in the extravascular PDAC stroma with significant co-localization with CAFs in the iRGD group (Fig. 5e). The QDs were also found around PanINs suggesting that the method could detect premalignant lesions. Only trace QD signals were noted in non-tumor tissues (Supplementary Fig. 4a). Collectively, the etchable QD system in combination with iRGD can serve as an intravenous probe to detect tumors, especially desmoplastic tumors, with high specificity.

Intraperitoneal delivery of ZHS-QDs to peritoneal tumors. Intraperitoneally administered iRGD facilitates penetration of co-administered compounds into peritoneal tumors in a tumor- specific manner ${ }^{33}$. Accordingly, the ZHS-QDs in combination with iRGD were tested for the ability to probe peritoneal tumors created with luciferase-positive MKN45P-luc human gastric cancer cells in mice ${ }^{33}$. Intraperitoneal co-injection of iRGD and ZHS-QDs led to strong fluorescence localized in the abdomen of the peritoneal tumor mice (Fig. 6a). No obvious extraperitoneal signal was noted. Subsequent intraperitoneal injection of Ag-TS abolished most of the fluorescence within 5 min leaving a focal signal, which co-localized with luminescent peritoneal tumors. The peritoneal tumor-specific probing was confirmed by in situ imaging of the mice (Fig. 6a) and ex vivo imaging of resected tissues (Fig. 6a). Signal intensity in the resected peritoneal tumors was threefold higher in the iRGD group than those in the control group (Fig. 6b). Confocal microscopy showed strong QD signals in peritoneal tumors especially in stroma-rich areas (Fig. 6c). Almost no QDs were found in non-tumor tissues (Supplementary Fig. 4b). Intraperitoneal injection of ZHS-QDs and Ag-TS without iRGD did not lead to tissue probing.

The lack of systemic NIR signals in the peritoneal tumor mice that received iRGD and ZHS-QDs suggested that the QDs entered the tumors predominantly through local penetration and not through the circulation. Indeed, intraperitoneal co-administration of iRGD promotes local entry of dextran and small-molecule drugs into peritoneal tumors in a circulation-independent manner ${ }^{33}$. However, iRGD-coated nanoparticles have been shown to enter the circulation from the abdominal cavity, and target peritoneal tumors through a combination of both circulation-dependent and -independent pathways $^{34,35}$. To separate the two pathways in the peritoneal tumor targeting by iRGD/ZHS-QDs, we tested the efficacy of extraperitoneal tumor targeting by intraperitoneally administered 
a

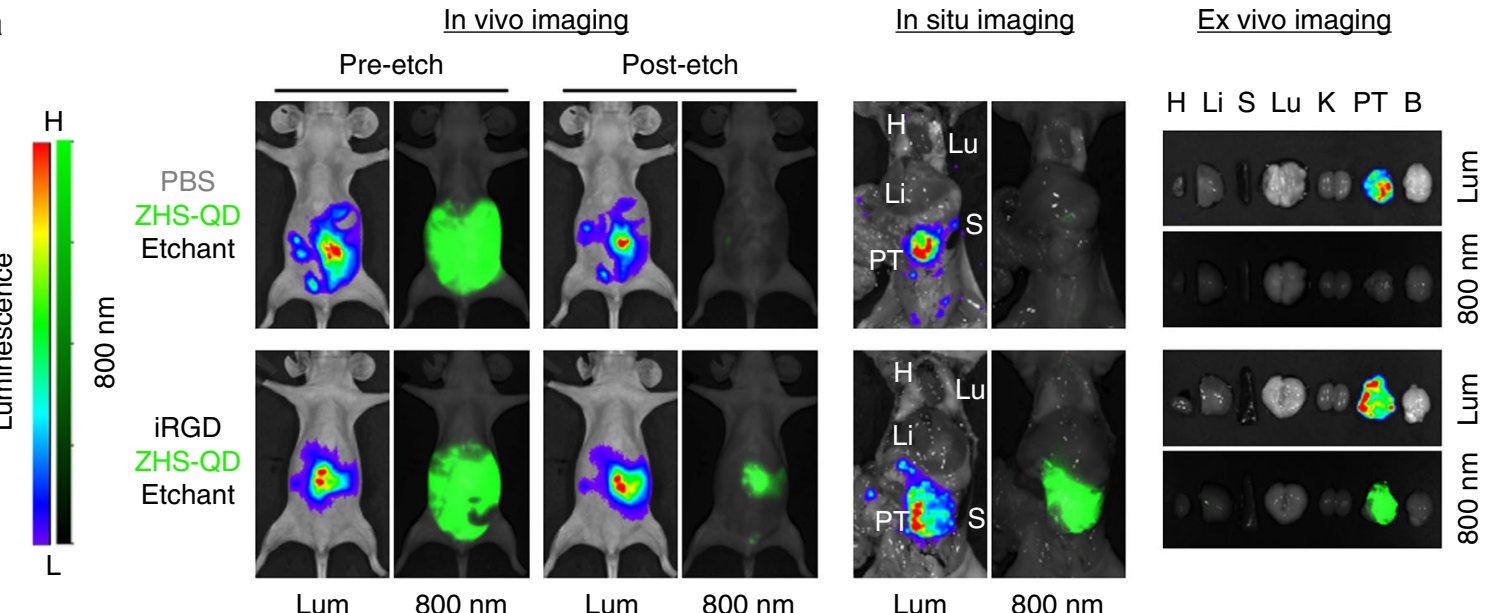

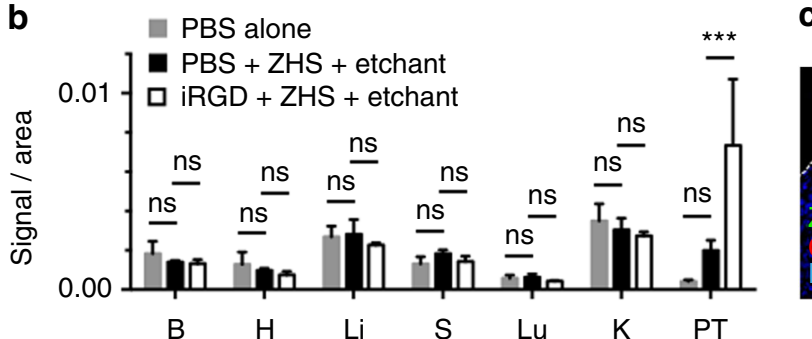

d

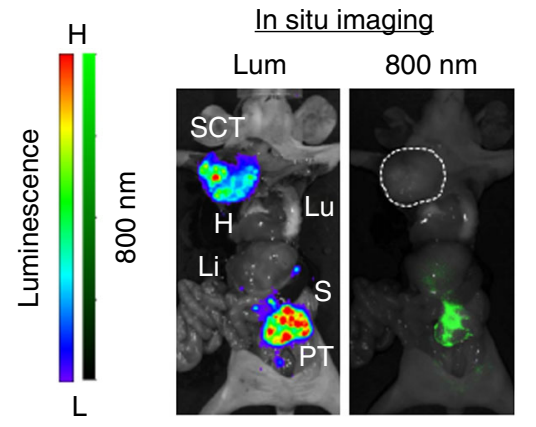

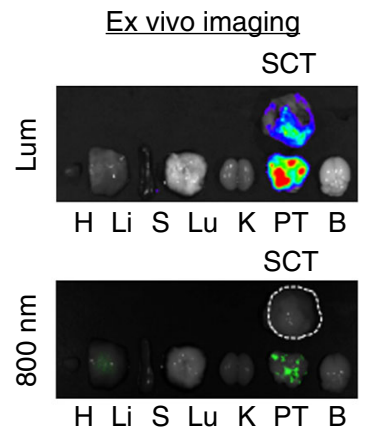

C
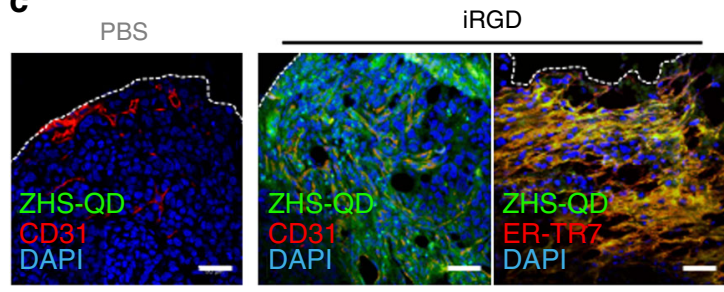

e

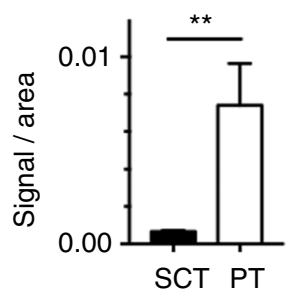

Fig. 6 Peritoneal tumor imaging with ZHS-QDs. Mice bearing peritoneal tumors (PTs) created with MKN45P-luc luciferase-positive human gastric cancer cells received an intraperitoneal co-injection of iRGD or PBS with ZHS-QDs. Intraperitoneal etchant (1x Ag-TS) was given 90 min later. $n=3$ per group. Some mice carried asubcutaneous MKN45P-luc tumor (SCT) in addition to the PTs. a, $\mathbf{d}$ Whole body in vivo and post-necropsy in situ imaging of the tumor mice, and ex vivo imaging of resected tissues. Lum, luminescence; $800 \mathrm{~nm}$, NIR. The white dotted line in $\mathbf{d}$ marks a SCT in a mouse that received iRGD, ZHSQDs, and Ag-TS. b, e Fluorescent signal per unit area in collected tissues $\mathbf{b}$ and in SCT vs. PT in the iRGD group e. c Confocal micrographs of PTs. Blue, DAPI; red, CD31 or ER-TR7; green, ZHS-QDs; scale bars, $50 \mu \mathrm{m}$. The white dotted lines indicate PT surface. B, brain; H, heart; Li, liver; S, spleen; Lu, lung; K, kidney. Statistics, two-way analysis of variance $\mathbf{b}$ or Student's $t$-test $\mathbf{e}$; error bars, SEM; ns, not significant; ${ }^{\star \star} P<0.01$; ${ }^{\star \star \star} P<0.001$

iRGD/ZHS-QDs in mice bearing a subcutaneous MKN45P-luc tumor in addition to the peritoneal tumors. Intraperitoneal injections of iRGD and ZHS-QDs followed by Ag-TS led to strong NIR signals in the peritoneal tumors, while the subcutaneous tumor remained macroscopically unprobed (Fig. 6d). Quantification of fluorescence intensity in the resected subcutaneous and peritoneal tumors further confirmed the results (Fig. 6e). These results strongly suggest that the ZHS-QDs minimally entered the circulation, and if any, circulating ZHSQDs had minor effects on peritoneal tumor targeting after intraperitoneal co-injection of iRGD and ZHS-QDs. Thus, the peritoneal tumor-specific probing was likely a result of local QD entry into the tumors facilitated by iRGD. These results indicate that local delivery of etchable QDs into peritoneal tumors followed by etching of excess QDs in the abdominal cavity allows peritoneal tumor-specific imaging. Collectively, the etchable QD system in combination with iRGD can be a powerful diagnostic platform and an aid in debulking surgeries for peritoneal carcinomatosis 33,36 , in addition to serving as an intravenous imaging probe for various types of tumors.

Synthesis and characterization of $\mathbf{H g}$-free etchable QDs. Given the success in highly tumor-specific imaging using the etchable ZHS-QD system, we set out to improve the system by synthesizing $\mathrm{Hg}$-free PEGylated QDs to avoid any potential $\mathrm{Hg}$ exposure in vivo. The QDs, ZAS-QDs, consisted of $\mathrm{Zn}, \mathrm{Ag}$, Se and $\mathrm{S}$, as confirmed by EDS analysis (Supplementary Table 1). The core diameter of the ZAS-QDs was $6.8 \pm 4.3 \mathrm{~nm}$ based on TEM measurements (Fig. 7a and Supplementary Fig. 5a). The $\mathrm{PL}$ emission extended beyond $800 \mathrm{~nm}$ with a peak at $708 \mathrm{~nm}$, 
a

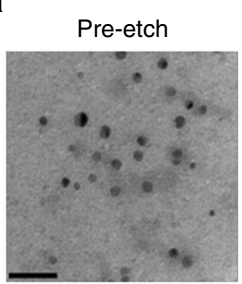

Post-etch

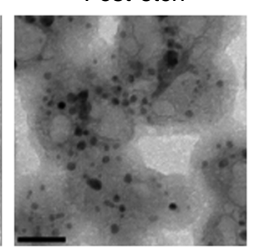

b

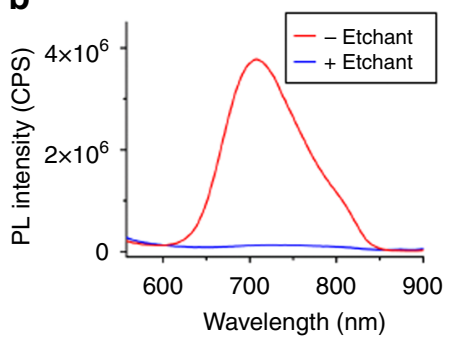

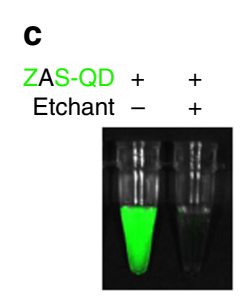

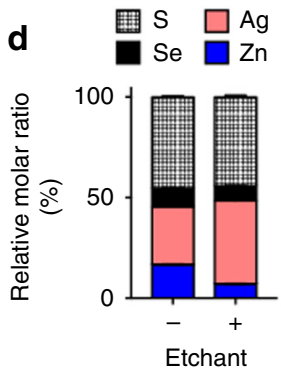

e
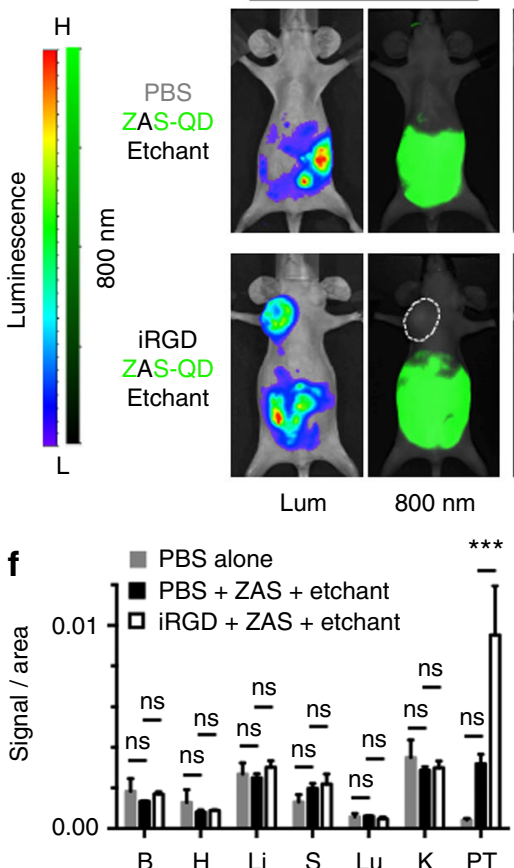

In vivo imaging

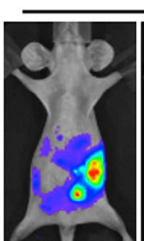

Pre-etch
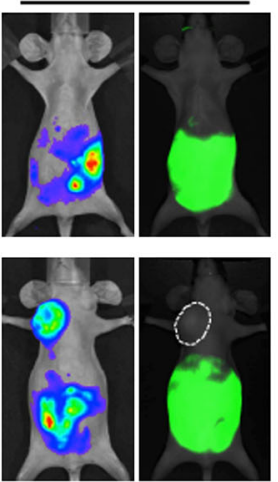

$800 \mathrm{~nm}$

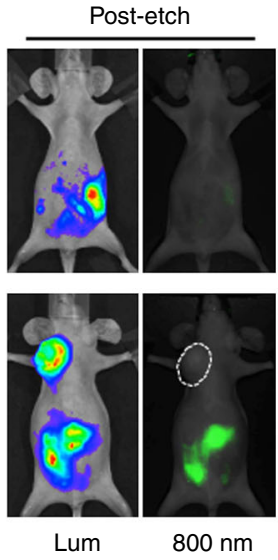

In situ imaging
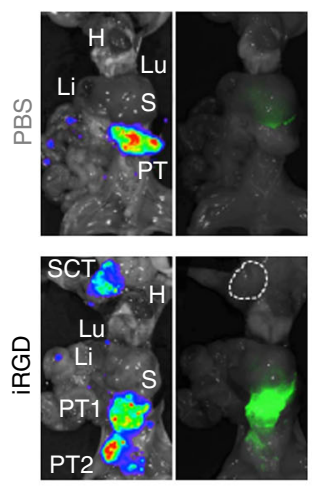

Lum $\quad 800 \mathrm{~nm}$

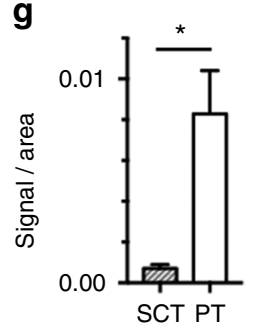

h
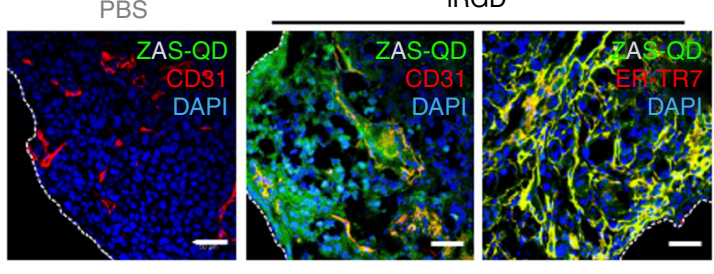

Fig. 7 Peritoneal tumor imaging with Hg-free etchable ZAS-QDs. a-c ZAS-QDs characterized by TEM a, PL spectra (excitation: $460 \mathrm{~nm}$ ) b and NIR imaging with a Li-Cor Pearl imager (green, ZAS-QDs) $\mathbf{c}$ before and after Ag-TS treatment. Scale bars, $50 \mathrm{~nm}$. d EDS analysis of ZAS-QDs before and after Ag-TS treatment showed relatively constant $S$ and Se and increased Ag after etching. See Supplemental Table 1 for complete data set. $n=3$ per group. e-h Mice bearing peritoneal tumors (PTs) created with MKN45P-luc luciferase-positive human gastric cancer cells received intraperitoneal co-injection of iRGD or PBS with ZAS-QDs followed by Ag-TS. $n=3$ per group. In e, live in vivo and post-necropsy in situ images of the tumor mice, and ex vivo images of resected tissues are shown. Lum, luminescence; $800 \mathrm{~nm}$, NIR. The white dotted line marks a subcutaneous tumor (SCT).NIR signal per unit area in collected tissues $\mathbf{f}$ and in SCT vs. PT in the iRGD group $\mathbf{g}$. B, brain; H, heart; Li, liver; S, spleen; Lu, lung; K, kidney. Statistics, two-way analysis of variance f or Student's t-test $\mathbf{g}$; error bars, SEM; ns, not significant; ${ }^{\star} P<0.05$; ${ }^{\star \star \star} P<0.001$. Confocal micrographs of PTs h. Blue, DAPI; red, CD31 or ER-TR7; green, ZAS-QDs; scale bars, $50 \mu \mathrm{m}$; white dotted lines in $\mathbf{h}$, PT surface

which made the QDs applicable to NIR imaging (Fig. 7b, c, and Supplementary Fig. 5b). The QY was $2 \%$.

Ag-TS abolished the PL in seconds in vitro, and slightly reduced the core size to $5.2 \pm 1.8 \mathrm{~nm}$ (Fig. $7 \mathrm{a}-\mathrm{c}$, Supplementary Table 2 and Supplementary Fig. 5a). Elemental analysis showed that the $\mathrm{Ag}$ to $\mathrm{Zn}$ ratio significantly increased upon $\mathrm{Ag}$-TS treatment without major changes in the anion ratio indicating that cation exchange took place (Fig. $7 \mathrm{~d}$ and Supplementary Fig. 5c). Nearly $90 \%$ of $\mathrm{C}$ and $\mathrm{O}$ remained after etching indicating that the PEG layer largely remained associated with the QD surface (Supplementary Table 1). Crystallographic analysis with XRD of the QDs showed a peak shift towards a higher angle with the emergence of peaks for $\mathrm{Ag}_{2} \mathrm{~S}$ and/or $\mathrm{Ag}_{2} \mathrm{Se}$ after $\mathrm{Ag}$-TS treatment, supporting the mechanism of quenching by $\mathrm{Ag}$ ion exchange in the QD core (Supplementary Fig. 5d).

In vivo imaging of peritoneal tumors with ZAS-QDs. The ZASQDs were tested in mice bearing MKN45P-luc peritoneal tumors for in vivo imaging. Intraperitoneally injected ZAS-QDs were strongly detected in live animals with a Li-Cor Pearl imager under an $800 \mathrm{~nm}$ channel (Fig. 7e). The NIR signal was limited to the abdominal cavity suggesting minimal entry of ZAS-QDs into systemic circulation. Subsequent intraperitoneal injection of Ag-TS eliminated the signal within 5 min. Almost no fluorescence was noted in peritoneal tumors suggesting that there was minimal tumor entry of the QDs. When iRGD was given together with the ZAS-QDs and subsequent intraperitoneal etching was performed, strong fluorescent signals co-localizing with luminescent peritoneal tumors were found. In situ imaging of abdominal and thoracic organs and ex vivo imaging of resected tissues (Fig. 7e) confirmed the peritoneal tumor specificity of the NIR signals. Almost no NIR signal was noted in extraperitoneal subcutaneous tumors suggesting that the ZAS-QDs entered the peritoneal tumors through a circulation-independent fashion (Fig. $7 \mathrm{e}-\mathrm{g}$ ). Consistent with these findings, confocal microscopy showed strong QD signals on peritoneal tumor surfaces with 

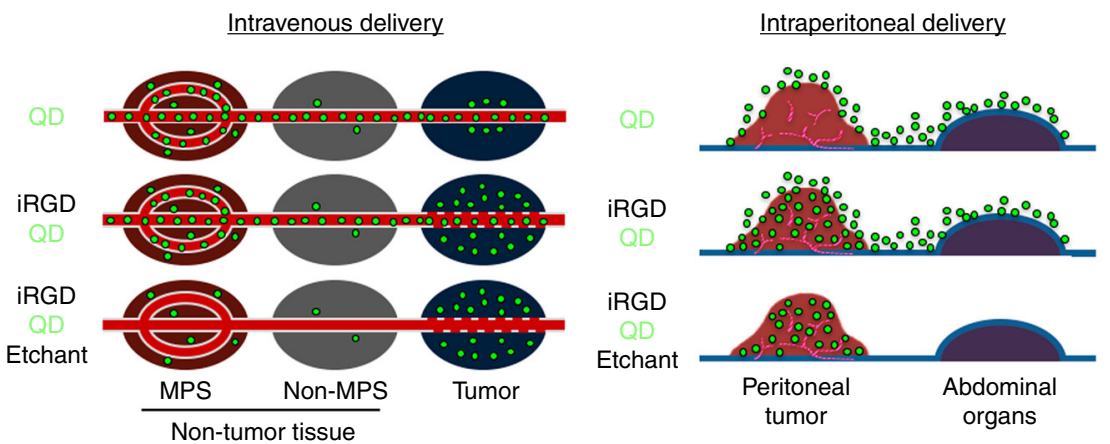

Fig. 8 Tumor-specific imaging with etchable QDs. (Intravenous delivery) Schematic of tumor-specific imaging with tumor-penetrating etchable QDs delivered through the circulation. The schematic depicts an early post-etching time point. Top panel: QDs (green particles), when injected alone, eventually accumulate mainly in the MPS by passing through the rich sinusoids. Some QDs may enter the extravascular tumor tissue because of the EPR effect. Middle panel: Pre-injection of iRGD facilitates extravasation and cell internalization of QDs in a tumor-specific manner. The QDs also eventually accumulate in the MPS. Bottom panel: Etching performed soon after the iRGD-mediated QD delivery into extravascular tumor cells causes quenching of intravascular and extracellular QDs leading to highly tumor-specific signals. (Intraperitoneal delivery) Schematic of peritoneal tumor imaging with intraperitoneally delivered etchable QDs. Top panel: intraperitoneal QDs attach to the tumor and peritoneal surfaces. Middle panel: iRGD facilitates local penetration of QDs specifically into peritoneal tumors. Bottom panel: Intraperitoneal etching differentially quenches the QDs that did not enter the tumors without affecting the intratumoral QDs, leading to highly tumor-specific signals

decreasing signals extending into the tumor interior (Fig. 7h). The majority of the QD signals were associated with nucleated cells. Importantly, there was significant co-localization with a reticular fibroblast marker suggesting that the stroma, mainly consisting of fibroblasts, served as a conduit for QD penetration into the tumors. In subcutaneous tumors, some QD-positive blood vessels were present suggesting that the QDs entered the circulation to some extent at microscopic levels (Supplementary Fig. 4b). QDs were not evident in non-tumor tissues. Thus, the Hg-free ZAS-QDs have etchable properties similar to ZHS-QDs, and provide highly tumor-specific signals when used in combination with iRGD and the Ag-TS etchant, demonstrating their capacity in tumor-specific in vivo imaging.

\section{Discussion}

This study introduces an important concept in imaging whereby probe brightness is controlled in vivo with a biocompatible chemical reaction. We have achieved exceptionally tumor-specific imaging by delivering etchable QDs specifically into tumor tissue through intravenous and intraperitoneal routes followed by preferential etching of excess counterparts in non-tumor tissues (Fig. 8). The differential etching is enabled by the contrast of QD dynamics between the rapid and active intratumoral spreading facilitated by iRGD, and the slow and passive accumulation into normal tissues 5 , 37 . In particular, performing the etching procedure soon after achieving tumor-specific targeting of PEGylated QDs can minimize their non-specific entry into the MPS (e.g., liver and spleen). The lack of etching of intratumoral QDs can be attributed to a combination of endocytosis, which protects QDs from a membrane-impermeable etchant, and the transient time window for iRGD to deliver QDs into tumor tissue and cells before subsequent etching is performed ${ }^{21}$.Compared to our previous Ag nanoparticle-based system that releases a dye upon etching ${ }^{38}$, it is noteworthy here that low background was achieved by directly modifying the chemical composition of the emitting species-a concept that may be extended to other fluorophores and imaging modalities. Although a metal-free etchable platform with a dissolvable core is ultimately desired for human use, this proof-of-concept study lays the groundwork for next-generation nanoprobes with extremely high tumorspecificity.

\section{Methods}

Materials. Zinc acetate dihydrate $\left[\mathrm{Zn}(\mathrm{OAc})_{2} \cdot 2 \mathrm{H}_{2} \mathrm{O}, 99.9 \%\right]$, mercury(II) perchlorate hydrate $\left[\mathrm{Hg}\left(\mathrm{ClO}_{4}\right)_{2} \cdot \mathrm{xH}_{2} \mathrm{O}, 99.998 \%\right]$, poly(ethylene glycol) methyl ether thiol $\left(\mathrm{CH}_{3} \mathrm{O}-\mathrm{PEG} 2000-\mathrm{SH}, \mathrm{Mw} 2000\right)$, silver nitrate $\left(\mathrm{AgNO}_{3}, \geq 99.0 \%\right)$, sodium chloride $(\mathrm{NaCl})$, potassium chloride $(\mathrm{KCl}, \geq 99.0 \%)$, manganese(II) chloride tetrahydrate $\left(\mathrm{MnCl}_{2} \cdot 4 \mathrm{H}_{2} \mathrm{O}, \geq 99 \%\right)$, magnesium chloride $\left(\mathrm{MgCl}_{2}, \geq 98 \%\right)$, iron(III) chloride hexahydrate $\left(\mathrm{FeCl}_{3} \cdot 6 \mathrm{H}_{2} \mathrm{O}, 97 \%\right)$, potassium hexacyanoferrate (III) $\left[\mathrm{K}_{3} \mathrm{Fe}(\mathrm{CN})_{6}, \geq 99.0 \%\right]$, potassium hexacyanoferrate(II) trihydrate $\left[\mathrm{K}_{4} \mathrm{Fe}\right.$ $(\mathrm{CN})_{6} \cdot 3 \mathrm{H}_{2} \mathrm{O}, 98.5-102.0 \%$ ], sodium borohydride $\left(\mathrm{NaBH}_{4}, 99 \%\right)$,sodium thiosulfate pentahydrate $\left(\mathrm{Na}_{2} \mathrm{~S}_{2} \mathrm{O}_{3} \cdot 5 \mathrm{H}_{2} \mathrm{O}, \geq 99.5 \%\right)$, copper(II) sulfate pentahydrate $\left(\mathrm{CuSO}_{4}\right.$ $5 \mathrm{H}_{2} \mathrm{O}, \geq 98.0 \%$ ), D-penicillamine (98-101\%) and sodium 2,3-dimercaptopropanesulfonate monohydrate (DMPS, 95\%) were purchased from Sigma-Aldrich (St. Louis, MO). Thiol PEG amine (HS-PEG2000- $\mathrm{NH}_{2}$, Mw2000) $\mathrm{HCl}$ salt was purchased from JenKem Technology USA (Plano, TX). Amphiphilic ligand HS- $\left(\mathrm{CH}_{2}\right)_{11}-\left(\mathrm{OCH}_{2} \mathrm{CH}_{2}\right)_{6}-\mathrm{NH}_{2}$ was purchased from ProChimia (Gdansk, Poland). Selenium powder ( -325 mesh, 99.5\%) was purchased from Alfa Aesar (Ward Hill, MA). Ammonium bicarbonate $\left(\mathrm{NH}_{4} \mathrm{HCO}_{3}\right)$ and 3-mercaptopropionic acid $(\geq 99.0 \%)$ were from Fisher Scientific (Pittsburgh, PA). Sodium hydroxide $(\mathrm{NaOH})$ pellets were bought from Amresco (Solon, OH). iRGD and CRGDC peptides were synthesized in house ${ }^{21} . \mathrm{H}_{2} \mathrm{O}$ was purified using a Milli-Q (EMD Millipore) system.

Cell lines and mouse models. MCF10CA1a human breast cancer cells $^{39}$, KRASInk mouse PDAC cells $s^{31}$, PC-3 human prostate cancer cells ${ }^{30}$, and MKN45P-luc luciferase-positive human gastric cancer cells ${ }^{33}$ were cultured in Dulbecco's modified Eagle's medium supplemented with $10 \%$ fetal bovine serum, $100 \mathrm{U} \mathrm{ml}^{-1}$ penicillin and $100 \mathrm{\mu g} \mathrm{ml}^{-1}$ streptomycin. The human cell lines were authenticated by the DNA Analysis Core Facility at the Sanford Burnham Prebys Medical Discovery Institute (La Jolla, CA) and the KRAS-Ink cell line was authenticated by DDC Medical (Fairfield, OH). All the cells were tested negative for mycoplasma contamination. Female BALB/c and athymic nude mice were both purchased from Harlan Laboratories (Indianapolis, IN). Orthotopic breast tumors were generated by injecting $2 \times 10^{6}$ MCF10CA1a cells into the mammary fat pad $^{39}$, and orthotopic PDAC tumors were generated by injecting $2 \times 10^{6} \mathrm{KRAS}$-Ink cells into the pancreas ${ }^{20}$ of female nude mice at 10 weeks of age. Peritoneal tumor mice were generated by injecting $10^{7}$ MKN45P-luc cells into the peritoneal cavity of female nude mice at 10 weeks of age ${ }^{33}$. All animal procedures were performed according to protocols approved by the Institutional Animal Care and Use Committee at Sanford Burnham Prebys Medical Discovery Institute.

Synthesis and characterization of QDs. ZHS-QDs were synthesized based on a previously reported method with modifications ${ }^{40}$. Specifically, $\mathrm{CH}_{3} \mathrm{O}-\mathrm{PEG} 2000-\mathrm{SH}$ powder $(360 \mathrm{mg})$ was added into $\mathrm{NH}_{4} \mathrm{HCO}_{3}$ solution $(2.4 \mathrm{ml}, 0.2 \mathrm{M}, \mathrm{pH}=12.4$ adjusted with $5 \mathrm{M} \mathrm{NaOH}$ ) and completely dissolved after short sonication. $\mathrm{Zn}$ $(\mathrm{OAc})_{2} \cdot 2 \mathrm{H}_{2} \mathrm{O}(900 \mu \mathrm{l}, 100 \mathrm{mM})$ and $\mathrm{Hg}\left(\mathrm{ClO}_{4}\right)_{2} \cdot \mathrm{xH}_{2} \mathrm{O}(270 \mu \mathrm{L}, 100 \mathrm{mM})$ were then added into the solution and quickly mixed, followed by immediate addition of freshly prepared sodium hydrogen selenide (NaHSe, $180 \mu \mathrm{l}, 250 \mathrm{mM})^{40}$. Adding NaHSe caused the reaction solution to turn dark instantly, indicating QD formation. After mixing in the dark at room temperature (RT) for $1 \mathrm{~h}$, the solution was moved to $4{ }^{\circ} \mathrm{C}$ for passivation for $48 \mathrm{~h}$. After centrifugation $(11,000 \times \mathrm{g}, 10 \mathrm{~min}$, $4{ }^{\circ} \mathrm{C}$ ), the supernatant containing dispersed QDs was collected, neutralized with 
sodium acetate-acetic acid buffer ( $\mathrm{pH}$ 5.0, $1 \mathrm{M}$ ), and purified six times in PBS by ultrafiltration with Ultra-4 $10 \mathrm{kDa}$ centrifugal filters (EMD Millipore, Darmstadt, Germany). After another centrifugation $\left(11,000 \times g, 10 \mathrm{~min}, 4^{\circ} \mathrm{C}\right)$, the supernatant was filtered with a $0.22 \mu \mathrm{m}$ syringe filter, and kept in the dark at $4^{\circ} \mathrm{C}$ until use.

Positively charged ZHS-QDs (ZHS- $\mathrm{NH}_{2}$ ) were prepared via the same procedure as above except that $\mathrm{CH}_{3} \mathrm{O}-\mathrm{PEG} 2000$-SH was replaced with HSPEG2000- $\mathrm{NH}_{2}$. Mn/ZnS QDs were synthesized as previously reported ${ }^{41}$. CdSe/ZnS core/shell QDs were purchased from eBioscience (San Diego, CA). InP/ZnS QDs and CIS/ZnS QDs, both coated with 3-mercaptopropionic acid, were bought from NNCrystal US Corporation (Fayetteville, AR). All the QDs were water-soluble. CdSe/ZnS QDs coated with cell-penetrating KCDGRPARPAR peptides (LifeTein, Somerset, NJ) were prepared according to the manufacturer's instructions associated with the eFluor $605 \mathrm{NC}$ sulfhydryl-reactive conjugation kit (eBioscience). CIS/ZnS-QDs coated with HS- $\left(\mathrm{CH}_{2}\right)_{11}-\left(\mathrm{OCH}_{2} \mathrm{CH}_{2}\right)_{6}-\mathrm{NH}_{2}$ were prepared as described earlier ${ }^{42}$.

ZAS-QDs were synthesized by first adding 3-mercaptopropionic acid (10 $\mu \mathrm{l})$ and $\mathrm{Zn}(\mathrm{OAc})_{2} \cdot 2 \mathrm{H}_{2} \mathrm{O}(75 \mu \mathrm{l}, 100 \mathrm{mM})$ to $\mathrm{NH}_{4} \mathrm{HCO}_{3}$ solution $(0.2 \mathrm{ml}, 0.2 \mathrm{M}$, $\mathrm{pH}=12.8$ adjusted with $5 \mathrm{M} \mathrm{NaOH}$ ). After vortexing for $30 \mathrm{~s}, \mathrm{CH}_{3} \mathrm{O}$-PEG2000-SH (45 mg) and DMPS (5.4 mg) in $\mathrm{H}_{2} \mathrm{O}\left(0.2 \mathrm{ml}, 4^{\circ} \mathrm{C}\right.$ ) were added to the solution, and the solution was vortexed again for $30 \mathrm{~s}$. Finally, NaHSe $(15 \mu \mathrm{l}, 250 \mathrm{mM})$ was added, followed by immediate addition of $\mathrm{AgNO}_{3}(75 \mu \mathrm{l}, 200 \mathrm{mM})$ and vortexing for $30 \mathrm{~s}$. After mixing in the dark at RT for $1 \mathrm{~h}$, the solution was moved to $4^{\circ} \mathrm{C}$ for overnight passivation. The solution was centrifuged $\left(11,000 \times g, 10 \mathrm{~min}, 4^{\circ} \mathrm{C}\right)$, and the supernatant was washed first in $\mathrm{H}_{2} \mathrm{O}$, then in PBS, by ultrafiltration with Amicon Ultra-4 centrifugal filters (NMWL $10 \mathrm{KDa}$ ) until the $\mathrm{pH}$ of the filtrate neutralized. After another centrifugation $\left(11,000 \times \mathrm{g}, 10 \mathrm{~min}, 4^{\circ} \mathrm{C}\right)$, the supernatant was passed through a $0.22 \mu \mathrm{m}$ syringe filter, and kept in the dark at $4^{\circ} \mathrm{C}$ until use. The concentrations of all ZHS-QDs and ZAS-QDs samples described in this study are expressed as $\mathrm{Zn}^{2+}$ concentration unless otherwise stated.

TEM was performed with a JEM-1200EX II electron microscope (JEOL, Tokyo, Japan) operating at $80 \mathrm{kV}$. Absorbance spectra were measured with a

DU800 spectrophotometer (Beckman Coulter, CA), and PL spectra were measured with a FluoroMax-4 spectrofluorometer (Horiba, Kyoto, Japan). QYs for ZHS- and ZAS-QDs were determined by using Rhodamine $6 \mathrm{G}$ as standard (QY $=95 \%$ in ethanol). The QY values for CdSe/ZnS-, InP/ZnS- and CIS/ZnS-QDs were acquired from the commercial source. EDS spectra were acquired using aXL30-SFEG UHR scanning electron microscope (FEI, Hillsboro, OR) equipped with a Silicon Drift Detector iXRFEDS analyzer (E2V, Chelmsford, Essex, UK), operating at $10 \mathrm{kV}$. XRD measurementswere performed on a MiniFlex II X-ray diffractometer (Rigaku, Tokyo, Japan) with $\mathrm{Cu}-\mathrm{Ka}$ radiation $(\lambda=0.15418 \mathrm{~nm})$ at $30 \mathrm{kV}$ and $15 \mathrm{~mA}$. ICP-OES measurement was performed with an Optima 3000 DV ICP-OES spectrometer (Perkin-Elmer, Waltham, MA). DLS was carried out with Malvern Zetasizer Nano ZS90 (Worcestershire, UK) to determine the hydrodynamic size of the QDs.

Synthesis of etchants and in vitro etching studies. Ag-TS was synthesized as follows. $\mathrm{AgNO}_{3}(0.08 \mathrm{mmol})$ was added into PBS $(500 \mu \mathrm{l})$ and mixed for $30 \mathrm{~s}$ forming a precipitate. $\mathrm{Na}_{2} \mathrm{~S}_{2} \mathrm{O}_{3}$ in $\mathrm{H}_{2} \mathrm{O}(0.2 \mathrm{M}, 1 \mathrm{ml})$ was then added to the solution and mixed for $5 \mathrm{~min}$ to allow the precipitate to dissolve. After filtration with a 0.22 $\mu \mathrm{m}$ syringe filter, a clear colorless solution was obtained $(0.05 \mathrm{M} \mathrm{Ag}$-TS stock solution, designated as ' $10 \times$ '). The solution was diluted 5 or 10 times with PBS to obtain a $2 \times$ or $1 \mathrm{x}$ Ag-TS solution, which was used on the same day for in vivo etching studies.

To study the principle of etching, ZHS-QDs or ZAS-QDs in PBS $(7.5 \mathrm{mM}, 350 \mu \mathrm{l})$ were mixed with either PBS $(100 \mu \mathrm{l})$ or 10x Ag-TS etchant $(100 \mu \mathrm{l})$ for $1 \mathrm{~h}$ at RT, followed by ultrafiltration in $\mathrm{H}_{2} \mathrm{O}$ with Amicon Ultra- 0.5 centrifugal filters (NMWL $10 \mathrm{kDa}$ ). The filtrates and concentrates were collected separately for ICP-OES measurement ${ }^{42}$. The collected concentrates were also subjected to TEM observation and PL spectra measurement.

Aqueous solutions of various chemicals were prepared to examine their etching capacity in vitro. The concentration was $1 \mathrm{mM}$ for all the chemicals except for $\mathrm{Na}_{2} \mathrm{~S}_{2} \mathrm{O}_{3}$ (Na-TS), which was $2.5 \mathrm{mM}$, and Ag-TS and Cu-TS, which were prepared by mixing $\mathrm{AgNO}_{3}(2 \mathrm{mM})$ or $\mathrm{CuSO}_{4}(2 \mathrm{mM})$ with an equal volume of Na-TS $(5 \mathrm{mM})$. Each chemical $(400 \mu \mathrm{l})$ was mixed with ZHS-QDs $\left(400 \mu \mathrm{l}, 0.5 \mathrm{mM}\right.$ of $\left.\mathrm{Zn}^{2+}\right)$ at RT for $1 \mathrm{~min}$. Immediately, the mixtures were imaged with a Li-Cor Pearl imager under the $800 \mathrm{~nm}$ channel, and the PL spectra of the mixtures were also immediately measured with an excitation wavelength of $450 \mathrm{~nm}$. Emission peak intensity was used to calculate relative PL intensity. A mixture of ZHS-QD solution $(400 \mu \mathrm{l}, 0.5 \mathrm{mM})$ and $\mathrm{H}_{2} \mathrm{O}(400 \mu \mathrm{l})$ was used as a positive control, the relative PL intensity of which was considered as $100 \%$.

To study time-dependent quenching kinetics of ZHS-QDs, the QDs in PBS ( $1 \mathrm{ml}$ ) with $\mathrm{Zn}$ concentration of $5 \mu \mathrm{M}$ was added into a cuvette. Fluorescence was collected at regular time intervals, showing a steady fluorescence signal. Ag-TS ( $2 \mathrm{mM}$ of $\mathrm{Ag}$ ) was then quickly mixed in the sample in the cuvette to a final Ag concentration of $5 \mu \mathrm{M}$. A Horiba FluoroMax-4 spectrofluorometer was used at $450 \mathrm{~nm}$ excitation ( $2 \mathrm{~nm}$ slit width) and $650 \mathrm{~nm}$ emission ( $5 \mathrm{~nm}$ slit width) with integration time of $0.5 \mathrm{~s}$ per point. To correct for background signal, the intensity of PBS using these settings was measured and subtracted from all data points. To study stoichiometric quenching kinetics, equation (2) was used for different molar ratios of $\mathrm{Ag}$ to $\mathrm{Zn}$ :

$$
\text { ZHS quenched }(\%)=100 \times\left(1-\frac{\text { PL intensity of ZHS-QD with Ag-TS }- \text { background }}{\text { PLintensity of ZHS-QD without Ag-TS - background }}\right)
$$

All the experiments were performed at room temperature.

To study the etchability of different QDs, aqueous solutions ( $400 \mu \mathrm{l}$ per solution) of $\mathrm{Mn} / \mathrm{ZnS}$ (Zn concentration: $10 \mathrm{mM}), \mathrm{InP} / \mathrm{ZnS}\left(0.25 \mathrm{mg} \mathrm{ml}^{-1}\right)$ and 3-mercaptopropionic acid-coated and HS- $\left(\mathrm{CH}_{2}\right)_{11^{-}}\left(\mathrm{OCH}_{2} \mathrm{CH}_{2}\right)_{6}-\mathrm{NH}_{2}$-coated CIS/ $\mathrm{ZnS}\left(0.5 \mathrm{mg} \mathrm{ml}^{-1}\right)$ QDs were added with an equal volume of $1 \mathrm{x} \mathrm{Ag-TS}$ or $\mathrm{H}_{2} \mathrm{O}$, while CdSe/ZnS (QD concentration: $3.2 \mathrm{nM}$ ) in $\mathrm{H}_{2} \mathrm{O}(400 \mu \mathrm{l})$ was added with an equal volume of $10 \times \mathrm{Ag}$-TS or $\mathrm{H}_{2} \mathrm{O}$. After mixing at RT for $5 \mathrm{~min}$, the mixtures were subjected to PL imaging and PL spectra measurement. PL imaging was performed with an UV/White light transilluminator LMW-20 (UVP, Upland, CA) for Mn/ZnS QDs, an Illuma tool Bright Light System LT-9900 (Lightools Research, Encinitas, CA) for CdSe/ZnS and InP/ZnS QDs and HS- $\left(\mathrm{CH}_{2}\right)_{11^{-}}$ $\left(\mathrm{OCH}_{2} \mathrm{CH}_{2}\right)_{6}-\mathrm{NH}_{2}$-coated CIS/ZnS QDs, and a Li-Cor Pearl imager under $700 \mathrm{~nm}$ channel for 3-mercaptopropionic acid-coated CIS/ZnS QDs.

In vitro QD uptake by human tumor cells. PC-3 cells, which express neuropilin1 , a cell surface receptor for the KCDGRPARPAR peptide ${ }^{30}$, were incubated in 96-well plates with KCDGRPARPAR-coated CdSe/ZnS QDs (QD concentration: $25 \mathrm{nM}$ ) for $90 \mathrm{~min}$ at $37^{\circ} \mathrm{C}$. The cells were subjected to epifluorescence imaging with a Leica DMIRE2 microscope (Leica, Wetzlar, Germany) before and after the addition of $1 \mathrm{x} \mathrm{Ag-TS}(1 \mu \mathrm{l})$ to each well containing $100 \mu \mathrm{l}$ of culture media. MCF10CA1a cells were incubated with or without iRGD peptide (final concentration: $50 \mu \mathrm{M}$ ) in culture media in chambered coverglass (Nunc Lab-Tek II, Rochester, NY) for $30 \mathrm{~min}$ at $37^{\circ} \mathrm{C}$, and ZHS-QDs were added to each chamber (final concentration: $1 \mathrm{mM} \mathrm{Zn}{ }^{2+}$ ). After incubation for $2.5 \mathrm{~h}$ at $37^{\circ} \mathrm{C}$, the cells were washed once with PBS, and cultured in fresh culture media containing Hoechst $33342\left(10 \mu \mathrm{g} \mathrm{ml}^{-1}\right.$ in $400 \mu \mathrm{l}$, Molecular Probes, Eugene, OR) for $10 \mathrm{~min}$ at $37^{\circ} \mathrm{C}$. Etching was performed by adding $1 \mathrm{x} \mathrm{Ag-TS}(100 \mu \mathrm{l})$ to each chamber and incubating the cells for $1 \mathrm{~min}$ at RT. The cells were imaged with a Zeiss LSM 710 NLO confocal microscope (Carl Zeiss, Oberkochen, Germany) before and after etching.

Biodistribution, clearance, and in vivo toxicity. BALB/c female mice10-12 weeks of age were intravenously injected with $100 \mu \mathrm{L}$ of PBS or ZHS-QDs in PBS (18 nmol Zn per g body weight) followed 30 min later with intraperitoneal injection of $300 \mu \mathrm{L}$ of PBS or 1x Ag-TS. For biodistribution and clearance studies, the mice were sacrificed under deep anesthesia $1 \mathrm{~h}, 24 \mathrm{~h}$, and 10 days after Ag-TS injection. Major tissues, serum, feces and urine samples were collected for quantification of mercury with ICP-OES ${ }^{42}$. To examine in vivo toxicity, blood was collected from the retro-orbital plexus of each mouse under deep anesthesia into a lithiumheparin $1.3 \mathrm{ml}$ micro tube (Sarstedt, Nümbrecht, Germany) after $24 \mathrm{~h}$ and 1 week, and plasma was isolated by centrifugation. The plasma $(100 \mu \mathrm{l})$ was pipetted into a Comprehensive Diagnostic Profile Rotor (Product Part No. 500-1038, Abaxis, Union City, CA) and subjected to biochemical toxicity assays using an AbaxisVetScan VS2 chemistry analyzer. The mice were then sacrificed under deep anesthesia, and tissues were collected and processed for paraffin embedding. Sectioned paraffin blocks were stained with hematoxylin and eosin (H\&E), and whole slide scanning was performed with a Leica SCN 400 slide scanner.

Imaging of normal mice. Female nude mice without tumors were anesthetized with isoflurane and imaged with an $800 \mathrm{~nm}$ channel with a Pearl Impulse small animal imaging system (Li-Cor, Lincoln, NE) before any injection. Then, the mice were intravenously injected with ZHS-QDs at a dose of $18 \mathrm{nmol} \mathrm{Zn} \mathrm{per} \mathrm{g} \mathrm{body-}$ weight per injection. Some mice received an intravenous dose of PBS $(100 \mu \mathrm{l})$ or iRGD (100 $\mu \mathrm{l}, 2.5 \mathrm{mM}$ in PBS) $25 \mathrm{~min}$ before the QD injection. Subsequent etching was performed at time points indicated in each Figure by either anintravenous injection of $1 \mathrm{x} \mathrm{Ag-TS}(100 \mu \mathrm{l})$, intraperitoneal injection of $1 \times \mathrm{Ag}$-TS $(300 \mu \mathrm{l})$, or three consecutive subcutaneous injections of a mixture of D-penicillamine and $\mathrm{CuSO}_{4}$ at a volume of $50 \mu \mathrm{l}$ per injection. The penicillamine/ $\mathrm{CuSO}_{4}$ solution was prepared by mixing stock solutions of D-penicillamine $(40 \mathrm{mM})$ and an equal volume of $\mathrm{CuSO}_{4}(20 \mathrm{mM})$ just prior to use and without filtration. We used a higher intraperitoneal dose of Ag-TS than that of intravenous $\mathrm{Ag}$-TS because intraperitoneal delivery generally leads to lower peak plasma concentrations than intravenous delivery ${ }^{13}$. In some cases, the mice were sacrificed under deep anesthesiafor tissue collection.

Imaging of breast tumors and PDAC in mice. Pre-injection imaging of mice bearing an orthotopic MCF10CAla breast tumor or KRAS-Ink PDAC was performed under anesthesia with isoflurane using a Pearl Impulse imager equipped with an $800 \mathrm{~nm}$ channel. The mice were intravenously injected with PBS (100 $\mu \mathrm{l})$ or iRGD or CRGDC in PBS $(100 \mu \mathrm{l}, 2.5 \mathrm{mM})$, followed by an intravenous injection of ZHS-QDs in PBS $(100 \mu \mathrm{l})$ at a dose of $18 \mathrm{nmol} \mathrm{Zn}$ per g body-weight $25 \mathrm{~min}$ later. Subsequent etching was performed by an intraperitoneal injection of $300 \mu \mathrm{l}$ of $1 \times \mathrm{Ag}$-TS in breast tumor mice or an intravenous injection of $100 \mu \mathrm{l}$ of $2 \mathrm{x} \mathrm{Ag-TS}$ in PDAC mice. In some cases, ZHS- $\mathrm{NH}_{2}$ QDs in PBS $(100 \mu \mathrm{l})$ at a dose of $18 \mathrm{nmol}$ 
Zn per g body-weight were injected instead of ZHS-QDs. The mice were anesthetized and imaged at different time points as shown in each Figure. After in vivo imaging, the mice were sacrificed under deep anesthesia. Breast tumor mice received cardiac perfusion of PBS, while PDAC mice did not. The necropsied mice (in situ imaging) and resected tissues (ex vivo imaging) were imaged with the Pearl Impulse small animal imaging system. The tissues were processed for immunofluorescence as described elsewhere ${ }^{20}$. In some cases, PDAC tissues were embedded in paraffin for H\&E staining and subsequent histology analysis as described elsewhere ${ }^{21}$. Fluorescence intensity quantification was performed with Li-Cor Image Studio Lite 4.0 software to calculate CI, fluorescent signal per tissue area, and $\mathrm{T} / \mathrm{Li}$ ratios.

Imaging of peritoneal tumor in mice. Mice bearing MKN45P-luc peritoneal tumors were intraperitoneally injected with ZAS-QDs ( $30 \mathrm{nmol} \mathrm{Zn}$ per g bodyweight) or ZHS-QDs ( $45 \mathrm{nmol} \mathrm{Zn}$ per g body-weight) in PBS (500 $\mu \mathrm{l}$ ) with or without $450 \mu \mathrm{g}$ of iRGD. At 70 min postinjection of QDs, the mice were intraperitoneally injected with luciferin $\left(15 \mathrm{mg} \mathrm{ml}^{-1}\right.$ in PBS, Biosynth International, Itasca, IL) at a dose of $0.28 \mathrm{mg}$ per g body-weight. The mice were then anesthetized with isoflurane, and imaged at different time points for luminescence with a Xenogen IVIS imager (Perkin-Elmer) and for NIR with a Li-Cor Pearl Impulse imager under $800 \mathrm{~nm}$ channel. Some mice also had an extraperitoneal subcutaneous MKN45P-luc tumor in the presence of the peritoneal tumors. At 90 min postinjection of QDs, $1 \times$ Ag-TS $(400 \mu \mathrm{l})$ was intraperitoneally injected. After $5 \mathrm{~min}$, the mice were imaged with the Xenogen IVIS and Li-Cor Pearl Impulse imagers. The mice were then immediately sacrificed under deep anesthesia by cardiac perfusion with PBS, and the necropsied mice (in situ imaging) and resected tissues (ex vivo imaging) were imaged again. The abdominal cavity of the necropsied mice was not washed before in situ imaging. The tissues were processed for immunofluorescence as described elsewhere ${ }^{33}$. Fluorescence intensity quantification was performed with Li-Cor Image Studio Lite 4.0 software.

Immunofluorescence. Tissue sections were treated with $0.25 \%$ Triton X-100 for $10 \mathrm{~min}$, washed with PBS 3 times, blocked with $1 \%$ bovine serum albumin for $1 \mathrm{~h}$, and incubated with a rat anti-mouse CD31 primary antibody (Catalog number: 553370, BD Biosciences, San Jose, CA), rabbit anti-mouse $\alpha$-SMA antibody (Product code: ab5694, Abcam, Cambridge, MA), or a rat anti-mouse ER-TR7 antibody (Catalog number: sc-73355, Santa Cruz Biotechnology, Dallas, TX) at $4{ }^{\circ} \mathrm{C}$ overnight. Secondary antibodies were Alexa Fluor 488 goat anti-rat IgG (Catalog number: A-11006) and Alexa Fluor 488 goat anti-rabbit IgG (Catalog number: A-11034)(Thermo Fisher Scientific, Rockford, IL). After washing with PBS, sections were mounted in DAPI-containing mounting medium (Vector Laboratories, Burlingame, CA) and examined under a Zeiss LSM 710 NLO confocal microscope.

Statistical analysis. At least three mice were analyzed per group. The mice were randomly assigned to each study group. No sample size estimates or blinding techniques were used. All statistical analyses were conducted with GraphPad Prism 7 or Excel $\left({ }^{\star} P<0.05,{ }^{* *} P<0.01,{ }^{* * *} P<0.001\right.$; ns, not significant). Unless otherwise stated, all data are expressed as mean \pm standard error of the mean (SEM). Statistical analysis was performed using Student's two-tailed $t$-test or one-way or two-way analysis of variance followed by post hoc analysis. A value of $P<0.05$ was considered statistically significant.

Data availability. The authors declare that the data of this study are available within the article and its Supplementary Information, or from the corresponding authors upon reasonable request.

Received: 16 January 2017 Accepted: 2 June 2017

Published online: 24 August 2017

\section{References}

1. Choi, H. S. et al. Design considerations for tumour-targeted nanoparticles. Nat. Nanotechnol. 5, 42-47 (2010).

2. Barreto, J. A. et al. Nanomaterials: applications in cancer imaging and therapy. Adv. Mater. 23, H18-H40 (2011).

3. Yu, M. X. \& Zheng, J. Clearance pathways and tumor targeting of imaging nanoparticles. ACS Nano 9, 6655-6674 (2015).

4. Jokerst, J. V., Lobovkina, T., Zare, R. N. \& Gambhir, S. S. Nanoparticle PEGylation for imaging and therapy. Nanomedicine 6, 715-728 (2011)

5. Gao, J. H. et al. In vivo tumor-targeted fluorescence imaging using near-infrared non-cadmium quantum dots. Bioconjugate Chem. 21, 604-609 (2010).

6. Son, D. H., Hughes, S. M., Yin, Y. D. \& Alivisatos, A. P. Cation exchange reactions in ionic nanocrystals. Science 306, 1009-1012 (2004)
7. Beberwyck, B. J., Surendranath, Y. \& Alivisatos, A. P. Cation exchange: a versatile tool for nanomaterials synthesis. J. Phys. Chem. C 117, 19759-19770 (2013).

8. Choi, H. S. et al. Renal clearance of quantum dots. Nat. Biotechnol. 25, 1165-1170 (2007).

9. Hockett, J. R. \& Mount, D. R. Use of metal chelating agents to differentiate among sources of acute aquatic toxicity. Environ. Toxicol. Chem. 15, 1687-1693 (1996).

10. Yerram, P., Saab, G., Karuparthi, P. R., Hayden, M. R. \& Khanna, R. Nephrogenic systemic fibrosis: a mysterious disease in patients with renal failure-role of gadolinium-based contrast media in causation and the beneficial effect of intravenous sodium thiosulfate. Clin. J. Am. Soc. Nephrol 2, 258-263 (2007).

11. Chen, C., He, X. W., Gao, L. \& Ma, N. Cation exchange-based facile aqueous synthesis of small, stable, and nontoxic near-infrared $\mathrm{Ag}_{2} \mathrm{Te} / \mathrm{ZnS}$ core/shell quantum dots emitting in the second biological window. ACS Appl. Mater. Interf. 5, 1149-1155 (2013).

12. Lee, J. W. \& Hwang, C. S. White light emission from a colloidal mixture containing $\mathrm{ZnS}$ based nanocrystals: $\mathrm{ZnS}, \mathrm{ZnS}: \mathrm{Cu}$ and $\mathrm{ZnS}: \mathrm{Mn}$. Bull. Korean Chem. Soc. 35, 189-196 (2014).

13. Schade, D. S., Eaton, R. P., Friedman, N. \& Spencer, W. The intravenous, intraperitoneal, and subcutaneous routes of insulin delivery in diabetic man. Diabetes 28, 1069-1072 (1979).

14. Blanco, E., Shen, H. \& Ferrari, M. Principles of nanoparticle design for overcoming biological barriers to drug delivery. Nat. Biotechnol. 33, 941-951 (2015).

15. Boulding, J. E. \& Baker, R. A. The treatment of metal poisoning with penicillamine. Lancet. 270, 985 (1957).

16. Nomura, S. et al. PET imaging analysis with ${ }^{64} \mathrm{Cu}$ in disulfiram treatment for aberrant copper biodistribution in menkes disease mouse model. J. Nucl. Med. 55, 845-851 (2014).

17. Zalups, R. K. Molecular interactions with mercury in the kidney. Pharmacol. Rev. 52, 113-143 (2000).

18. Maeda, H., Fang, J., Inutsuka, T. \& Kitamoto, Y. Vascular permeability enhancement in solid tumor: various factors, mechanisms involved and its implications. Int. Immunopharmacol. 3, 319-328 (2003).

19. Heldin, C. H., Rubin, K., Pietras, K. \& Ostman, A. High interstitial fluid pressure - an obstacle in cancer therapy. Nat. Rev. Cancer. 4, 806-813 (2004).

20. Sugahara, K. N. et al. Tissue-penetrating delivery of compounds and nanoparticles into tumors. Cancer. Cell. 16, 510-520 (2009).

21. Sugahara, K. N. et al. Coadministration of a tumor-penetrating peptide enhances the efficacy of cancer drugs. Science 328, 1031-1035 (2010).

22. Pang, H. B. et al. An endocytosis pathway initiated through neuropilin-1 and regulated by nutrient availability. Nat. Commun. 5, 4904 (2014).

23. Pang, H. B. et al. A free cysteine prolongs the half-life of a homing peptide and improves its tumor-penetrating activity. J. Control Release 175, 48-53 (2014).

24. Åkerman, M. E., Chan, W. C. W., Laakkonen, P., Bhatia, S. N. \& Ruoslahti, E Nanocrystal targeting in vivo. Proc. Natl Acad. Sci. USA 99, 12617-12621 (2002).

25. Mancini, M. C., Kairdolf, B. A., Smith, A. M. \& Nie, S. M. Oxidative quenching and degradation of polymer-encapsulated quantum dots: new insights into the long-term fate and toxicity of nanocrystals in vivo. J. Am. Chem. Soc. 130, 10836-10837 (2008).

26. Smith, A. M., Duan, H. W., Mohs, A. M. \& Nie, S. M. Bioconjugated quantum dots for in vivo molecular and cellular imaging. Adv. Drug Deliver. Rev. 60 , 1226-1240 (2008).

27. Koivunen, E., Gay, D. A. \& Ruoslahti, E. Selection of peptides binding to the alpha-5-beta-1 integrin from phage display library. J. Biol. Chem. 268, 20205-20210 (1993).

28. Jiang, T. et al. Tumor imaging by means of proteolytic activation of cellpenetrating peptides. Proc. Natl Acad. Sci. USA 101, 17867-17872 (2004).

29. Baish, J. W. et al. Scaling rules for diffusive drug delivery in tumor and normal tissues. Proc. Natl Acad. Sci. USA 108, 1799-1803 (2011).

30. Teesalu, T., Sugahara, K. N., Kotamraju, V. R. \& Ruoslahti, E. C-end rule peptides mediate neuropilin-1-dependent cell, vascular, and tissue penetration. Proc. Natl Acad. Sci. USA 106, 16157-16162 (2009).

31. Aguirre, A. J. et al. Activated Kras and Ink4a/Arf deficiency cooperate to produce metastatic pancreatic ductal adenocarcinoma. Genes Dev. 17, 3112-3126 (2003).

32. Mitchem, J. B. et al. Targeting tumor-infiltrating macrophages decreases tumorinitiating cells, relieves immunosuppression, and improves chemotherapeutic responses. Cancer Res. 73, 1128-1141 (2013).

33. Sugahara, K. N. et al. A tumor-penetrating peptide enhances circulationindependent targeting of peritoneal carcinomatosis. J. Control Release 212, 59-69 (2015).

34. Simón-Gracia, L. et al. Paclitaxel-loaded polymersomes for enhanced intraperitoneal chemotherapy. Mol. Cancer Ther. 15, 670-679 (2016). 
35. Simón-Gracia, L. et al. iRGD peptide conjugation potentiates intraperitoneal tumor delivery of paclitaxel with polymersomes. Biomaterials. 104, 247-257 (2016).

36. Sugarbaker, P. H. Cytoreductive surgery and hyperthermic intraperitoneal chemotherapy in the management of gastrointestinal cancers with peritoneal metastases: progress toward a new standard of care. Cancer Treat. Rev. 48, 42-49 (2016).

37. Wu, X., Tian, F., Zhao, J. X. \& Wu, M. Evaluating pharmacokinetics and toxicity of luminescent quantum dots. Expert Opin. Drug Metab. Toxicol. 9, 1265-1277 (2013)

38. Braun, G. B. et al. Etchable plasmonic nanoparticle probes to image and quantify cellular internalization. Nat. Mater. 13, 904-911 (2014).

39. Santner, S. J. et al. Malignant MCF10CA1 cell lines derived from premalignant human breast epithelial MCF10AT cells. Breast Cancer Res. Treat. 65, 101-110 (2001).

40. He, X. W., Gao, L. \& Ma, N. One-step instant synthesis of protein-conjugated quantum dots at room temperature. Sci. Rep. 3, 2825 (2013).

41. Yin, N. Q. et al. Preparation and characterization of nontoxic magneticluminescent nanoprobe. Chinese Phys. B 21, 116101 (2012).

42. Liu, X. Y. et al. Tumor-targeted multimodal optical imaging with versatile cadmium-free quantum dots. Adv. Funct. Mater. 26, 267-276 (2016).

\section{Acknowledgements}

This work was supported by grants R01CA167174 (K.N.S.), R01CA152327 (E.R.), R01CA188883 (E.R.), and P30CA030199 (CancerCenterSupportGrant, Sanford Burnham Prebys Medical Discovery Institute) from the National Cancer Institute of NIH, Career Development Award from American Association of Cancer Research/Pancreatic Cancer Action Network (PanCAN) (K.N.S.), and Translational Research Grant from PanCAN (K.N.S.).We thank Dr. VenkataRamanaKotamraju (Sanford Burnham Prebys Medical Discovery Institute) for peptide synthesis, Dr. Douglas Hanahan (Swiss Federal Institute of Technology Lausanne) for kindly providing the KRAS-Ink cells, and Dr. Kenneth Olive, Stephen Sastra, and Carmine Palermo (Columbia University College of Physicians and Surgeons) for their assistance with the animal work. We also thank Dr. Paterno Castillo (Scripps Institution of Oceanography, University of California, San Diego) for his assistance with the ICP-OES studies.

\section{Author contributions}

X.L., G.B.B. and K.N.S. designed the experiments. X.L. performed most of the experiments. M.Q. performed ICP-OES, EDS, and XRD measurements. X.L., G.B.B. and K.N.S wrote the manuscript. G.B.B., E.R., and K.N.S. supervised the study and edited the manuscript.

\section{Additional information}

Supplementary Information accompanies this paper at doi:10.1038/s41467-017-00153-y.

Competing interests: E.R. and K.N.S. have ownership interest (including patents) of DrugCendRInc, which holds a license to the iRGD peptide. The remaining authors declare no competing financial interests.

Reprints and permission information is available online at http://npg.nature.com/ reprintsandpermissions/

Publisher's note: Springer Nature remains neutral with regard to jurisdictional claims in published maps and institutional affiliations.

(c) (i) Open Access This article is licensed under a Creative Commons Attribution 4.0 International License, which permits use, sharing, adaptation, distribution and reproduction in any medium or format, as long as you give appropriate credit to the original author(s) and the source, provide a link to the Creative Commons license, and indicate if changes were made. The images or other third party material in this article are included in the article's Creative Commons license, unless indicated otherwise in a credit line to the material. If material is not included in the article's Creative Commons license and your intended use is not permitted by statutory regulation or exceeds the permitted use, you will need to obtain permission directly from the copyright holder. To view a copy of this license, visit http://creativecommons.org/ licenses/by/4.0/.

(C) The Author(s) 2017 